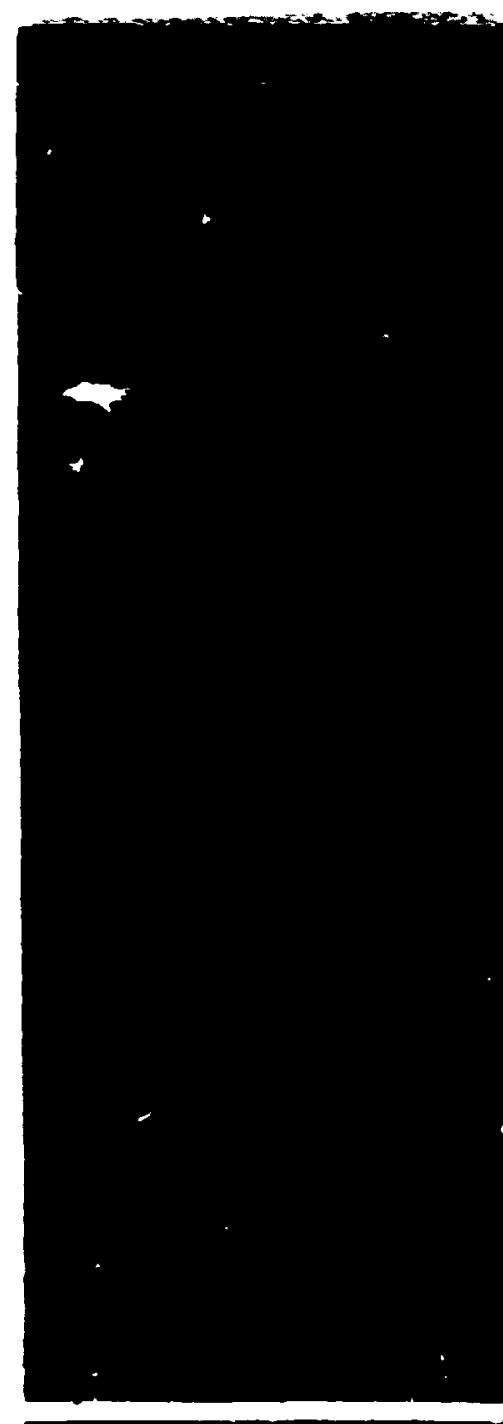

ORNL;CSD/TM-25

\title{
A NEW APPROACH TO SAFEGUARDS ACCOUNTING
}

D. H. Pike - G. W. Morrison

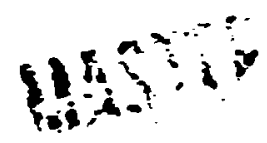


Printed in the United States of America. Available from National Technical information Service

U.S. Department of Commerce 5285 Port Royal Road. Springfield. Virginia 22161

Price: Printed Copy \$4.50; Microfiche $\$ 3.00$

This reoort was prepared as an account of work sponscred by the United Slates Government Neither the United States nor the Energy Hesearch and Devetopment Administration Unitod Slates Nuciear Regulatcry Commission, nor any of their employees. nor any of their contractors. subcontractors. or their employees. makes any warranty. express or implied. Or assumes any legal liability or responsibility for the accuracy. completeness or usefulness of iny intormation. apparatus. product or process disclosed. Or represents that its use would not infringe prwately owned righis 
ORericSO/TH-25

MRC Category 3

Contrac: No. -1405 eng 26

COMPUTER SCIENCES DIVISION

\title{
A NEL APPROACH TO SAFEGUARDS ACCOUNTING
}

D. H. Pike* and G. H. Morrison

\#IT!CE

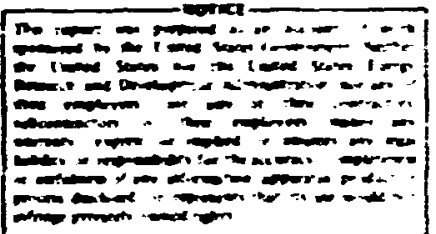

This document contains infcrmation of a preliminary nature.

It is subject to revision or correction and therefore does

not represent a firial report.

\author{
Date Published - March 1977 \\ Prepared for the \\ U.S. Nuclear Regulatory Commission \\ Office of Nuclear Material Safety \& Safeguards \\ Under Interagency Agreement ERDA 40-549-75
}

*Industrial Engineering Department, University of Tennessee, Knoxville

\author{
UNION CARBIOE CORPORATION, NUCLEAR DIVISION \\ operating the \\ Oak Ridge Gaseous Diffusion Plant - Oak Ridge Natiunal Laboratory \\ Oak Ridge $\mathrm{Y}-12$ Plant \\ for the \\ ENERGY RESEARCH AND DEVELOPMENT ADMINIST:SATIOII
}




\section{CONTENTS}

ABSTRICT ................................ 1

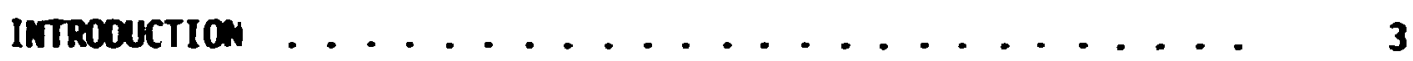

CURRERT ATPROACHES ...................... 5

KRUMN FILTER APPROACH ................... 10

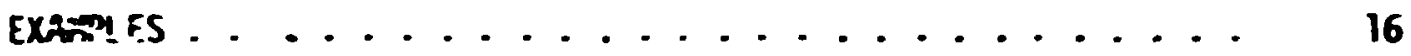

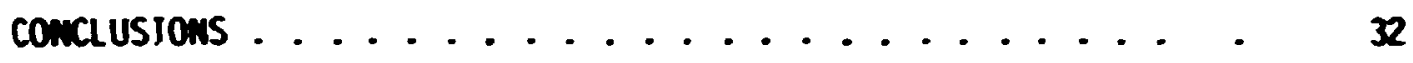

REFEREMCES ........................... 35 


\section{RBSTRACT}

In recent years there has been widespread concern over the problem of nuclear safeguards. Due to the proliferation of nuclear reactc 's throughout the worid, the concern about the loss or diversion of nuclear materials at various points in the fuel cycle has greatly increased. To minimize the possibility of material loss, the nuclear industry relies on Physical protection systems and materials accountability procedures at licensed facilities.

Present techniques of aterial accountability rely on double-entry accounting systems. For various reasons, only noisy observations of on-hand inventory are available. Hence one is forced to use statistical techniques in an attempt to detect the existence of missing material. Current practice is to use control charts as the basis for detecting significant material losses. Control charts may aid in detecting large material losses but are insensitive to small quantities of atel ial loss, even if these $\sin 11$ losses occur repeatedly over a long period of time.

The purpose of this research is to show the feasibility of using linear state estimation theory in nuclear material accountalility. The Kalman Filter is used as the state estimation: technique. The state vector wich consists of on-hand inventory and material losses is estimated recursively. 
The Kalman Filter approach is compared to the current statistical practices of MUF-LEWf control charts and Cusun charts. The simulated results show that in ascending order of sensitivity to material losses the techniques would be ranked as 1) MJF-LER.JP Control Charts 2) Cusin charts and 3) Kalman Filter. Not only is the Ralman Filter snown to be more sensitive to material losses, it is also shom to be a very accurate estimator of on-hand inventory. 


\section{INTROOUCTION}

Fissile material anj radioactive material are or will be present in the light-water eactor fuel cycle. Fissiie material is also present in nuclear weapon fabrication facilities. Possible loss of such material is a significant problen to the nuclear industry. To reduce the possibility of ioss of fissile and radioactive material, the nuclear industry relies on 1) physical security systems and 2) material accountability practices. Physical security measures serve as a deterrent to diversion of nuclear material. The material accountability procedures are designed "to deter the theft or civersion of these materials and to detect any such event if it occurs so that time!y recovery action can be taken" [1 pp. 517].

Detection is made difficult because of uncertainties in material balances in a "Material Balance Area" (MBA). Rosenbaum [2] and Jaech [3] disciss the sources of uncertainty. Basically, uncertainty occurs because of 1) human error (transposing figures when recording data, misreading instruments, failure to record data, etc. ', and 2) equipment error (incorrectly calibrated instruments, ma? functioning equipment, equipment accuracy and equipment limitations, etc.). One must, therefore, treat measurements of on-hand inventory and measurements of inputs and outputs of an MBA as random variables. Thus the froblem of detecting missing material is a statistical problem akin to the problem of recovering a signal from noisy data. 
There is also a problem of "timeliness of the data." Typically, an ma is closec at regular intervals. The data obtained from the closing together with historical data must be subjected to a statistical analysis to ascertain wether or not there is any loss of material. If it i; concluded that a loss has occurred, there is uncertainty as to whether the loss is cassed by missing material or inaccurate data. Hence, a recheck of fig.Jres is necessary. There is thus a sigrificant delay before an error is discovered and an assignable cause is determined. Use of computers, particularly real-time information systens, helps improve the timeliness of the data collection and data analysis.

Rosenbaum las proposed two classifications of material loss. Each of these is disc:ussed below:

1. One-tine loss of a large quantity of material:

Suzh a loss can be detected if the quantity stolen is sufficiently large. However, the detection will not occur until a closing occurs.

2. Continised loss of small quantities of material:

This type of loss is the most difficult to detect. The magnitudi? of uncertainty in the measurements results in existing accountability techniques being insensitive to smail quantity losses.

The review of current industry practice which follows attempts to summarize the current accountability practices and to explai:? why small quantity losses are difficult to detect using the:se practices.

\footnotetext{
Material Bal ance Closing refers to measuring inventcry, estimating hidden inventory, ecalling all inputs and outputs since the last clcsing, and estimating missing material.
} 


\section{CURRENT APPROACHES}

The standard industry practice has been sudiarized in the work of Jaech [3], [4], and [5]. A noisy estimate of muf is obtained at aterial balance closing:

$$
H(j)=Y(j)+H(j)-Y(j+1) \quad j \leq \vdots 1,2, \ldots, t
$$

where

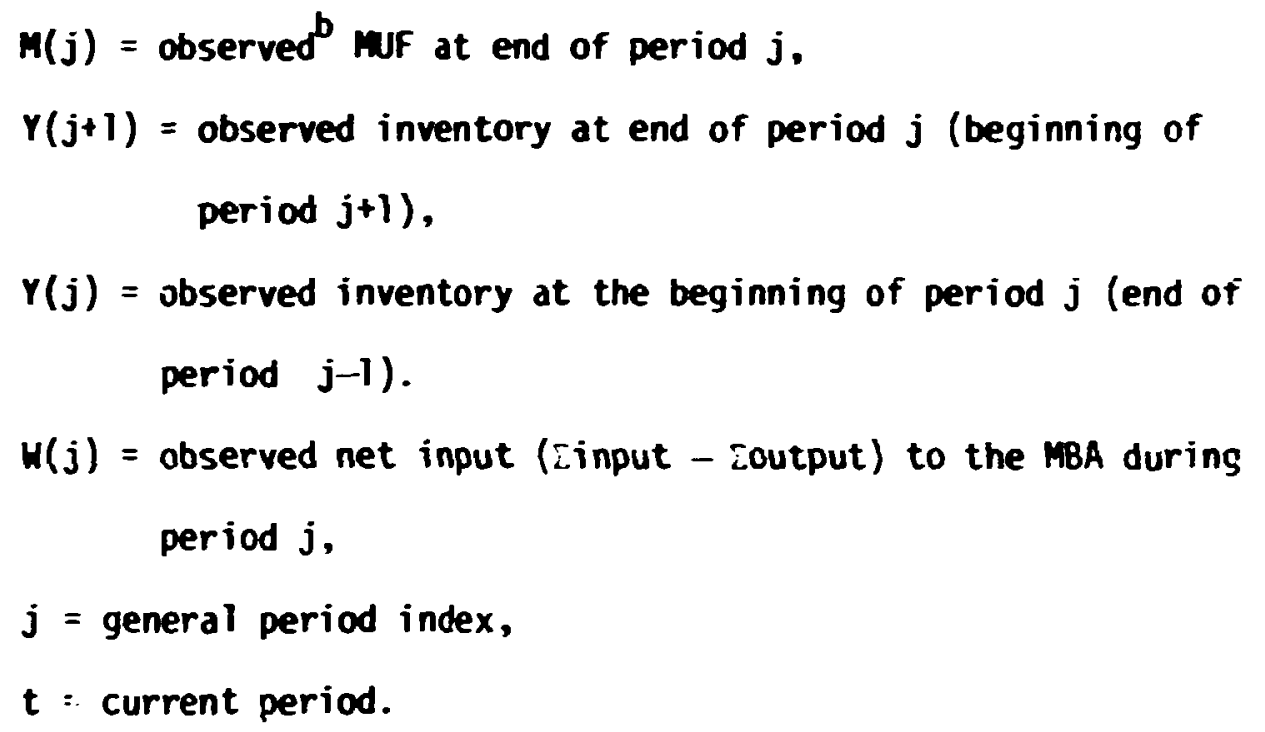

The measured inventory is assumed to consist of the true inventory, $I(j)$, plus a zero-mean random variable, $V(j)$, to account for measurement error:

$$
\begin{aligned}
& Y(j)=I(j)+V(j) \\
& E\left(V(j)^{\hat{j}}\right)=R(j) .
\end{aligned}
$$

Similarly, the net input, $W(j)$, is assumed to be composed of the true value, $T(j)$, plus a zero-meari measurement error

$$
H(j)=T(j)+Z(j)
$$

${ }^{b}$ observed values are composed of a true value and an additive zeromean random variable. 


$$
E\left(Z(j)^{2}\right)=S(j)
$$

Using these assumptions on measurement errors, and assuming that measurement errors are uncorrelated, it follows that the variance of $M(j)$ is:

$$
\sigma_{i}^{2}(j)=R(j+1)+R(j)+S(j)
$$

The procedure suggested by Jaech consists of using the variance in (6) to calculate a "limit of error for material undccounted for," (LEMJf):

$$
\text { LENF }= \pm 2 \sigma_{1}(j)
$$

A refinement on the concept of MF and LEmuf involves the use of control charts. The so-called "standard control chart" (SCC) involves the plotting of 1$)$ the time series $(M(j): j=1,2, \ldots, t j, 2)$ 2-sigma control limits and/or 3) 3-signa control limits. Control limits are usually of the form $\pm 2 \sigma_{1}(j)$. If we define an upper control limit, $U C L_{x}=+2 \sigma_{1}$, and a lower control limit, $L C L_{x}=-2 \sigma_{1}$, then if an otservation $M(j)$ falls within control limits:

$$
L C L_{x} \leq M(j) \leq U C L_{x} \quad j \varepsilon\{1,2, \ldots, t\},
$$

the process is assumed to be operating within the normal limits. If inequality $(8)$ does not hold for some $M(j)$, the variation in the observed MUF is assumed to be abrormal. In the terminology of statistical quality control, there is an "assigrable cause" for the variation in $M(j)$ when (8) ir violated. The use of 2-sigma control limits appears cormon but in some cases both two- and three-sigma control limits are used. The 2-sigma limits are warning limits while points outside the 3-sigma limits are assumed to be significant. Less than $1 \%$ of the points in a s.able 
process fall outside the 3-sigma limits. Points falling between the 2-sigma and 3-sigma limits may or may not be significant (up to 5: of the points in a stable process exhibit such behavior). The use of control charts enhances accountability practices since the analyst can look at the entire history of the time series rather than at a sing.e isolated point. Analysis of the history may disclose trends, abnormal patterns, etc., wich are indicative of material losses.

It has been noted [ $\underline{3}]$ and [E] that successive observed mf's exhibit serial correlation. This is due to the fact that the measured inventory, $Y(j)$, appears in the calculations for $M(j)$ and $M(j+1)$. This will result in a negative correlation even in a stable process. Thus, a large positive IUf is frequently follawed in the next period by a large negative MuF and vice versa. To take advantage of this knowledge, Jjech [3] proposes that a second control chart called the "difference control chart" (DCC) be ised in conjunction with the SCC. The quantity plotted is:

$$
O(j)=\left(M(j)-\mu_{m}\right)-\rho\left(M(j-1)-\mu_{M}\right) j \varepsilon\{2,3, \ldots, t\} .
$$

Control limits on the DCC are of the form:

$$
\pm 2 \sigma_{2}(t)
$$

where

$$
\sigma_{2}^{2}(t)=(1+\rho)^{2} R(t)+\rho^{2} R(t-1)+R(t+1)+Q(t)+\sigma^{2} Q(t-1) \text {. }
$$

The variable $\rho$, wich appears in equations $(10)$ and $(11)$, is the serial correlation coefficient of lag one. If $\rho=0$, the DCC provides the same information as the SCC. Johnson [6] has proposed accounting for serial 
correlation in the MUF data by the use of an SCC with $\sigma_{:}^{2}$ calculated using ar: equation based on a first-order autoregression process. There are several additional types of control charts which are currently in use. For example, the Cimulative Sum Control Chart (CUSUM Chart) piots at period $t$ :

$$
Z(t)=\sum_{j=1}^{t} M(j)
$$

The rontrol limits are: $\pm 2 \sigma_{3}(t)$

where

$$
\pi_{3}^{2}(t)=R(1)+R(t+1)+\sum_{i=1}^{t} Q(i) .
$$

Note that the control limits increase with increasing $t$. A variation on the CUSUM chart which results in control limits which decreases with increasing $t$ is the average CUSUM chart. On this chart is plotted:

$$
\overline{\bar{z}}(t)=\frac{!}{E} Z(t)=\frac{1}{t} \sum_{j=1}^{t} M(j)
$$

with control limits of the form: $\pm 2 r_{4}(t)$

where

$$
\sigma_{4}{ }^{2}(t)=\frac{1}{t^{2}}\left[R(1)+R(t+1)+\sum_{t=1}^{t} R(i)\right] .
$$

Jaech [3] has aiscussed a related statistical problem, that of estimating the on-nand inventory. Jaech suggests the use of a recursive estimation scheme to estimate on-hand inventory: 


$$
\Lambda(j+1)=a_{j} Y(j)+\left(1-a_{j}\right)[1(j)+W(j)] \quad j \in\{1,2, \ldots, t\}
$$

with $Z(0)=Y(0)$. The constant $\alpha_{j}$ in $(16)$ is chosen to minimize the variance of the estimate. $\Delta(j)$. Estimates of $M(j)$ can be obtained using:

$$
M(j)=\Delta(j)+H(j)-\Delta(j+1) \quad j \varepsilon\{1,2, \ldots t\} .
$$

The variance of $M(j)$ using (16) and (17) can be shown to be less than the variance of $M(j)$ using (i).

In sumary, the current nractice is to 1) use either equation (1) or equation (17) to calculate an observed MuF, $M(j) ; 2)$ calculate the differences $O(j)$, using equation $(9)$; 3) construct upper and lower control limits for observed MUF, $M(j)$, and control limits for the differerces, $D(j)$; and 4) construct a standard control chart, a difference control chart, or perhaps one of the CUSUM control charts. As long as points fall within the control limits, the variation in MUF is assumed to be varying within "normel" limits. This explains why current material accountability techniques can be expected to disciose large single losses but do not disclose small losses. If measurement errors result in observed MUF values varying randomly with a mean oi zero and a standard deviation of $\sigma_{1}$, the material losses can be detected only if the quantity of material lost is at least $3 \sigma_{1}$ (if 3-sigma control linits are used on a standard control chart). The CUSUM and average CUSUM charts are somewhat more sensitive than the SCC or DCC but also have an inherent difficulty in detecting small quantity losses. 
KALMAN FILTER APPROACH

The Kalman Filter approach which is developed in detail below offers several advantages over the traditioral control chart approach: these advantages are [7] and [8] j:

1. The Kalmen Filter uses the material balance equations and models of diversion in obtaining estimates.

2. The Kaiman Filter estimates "true material loss" as opposed to "MJF" used in the control chart approach.

3. The on-hand inventory and material loss are estimated simultaneously by the Kalman Filter. Thus, the interaction of those items of interest are accounted for through the model equations.

4. Measurement error is accounted for in the model.

5. Since more information is used in the estimation procedure, the Kalman filter produces estimates which are more sensitive to the repeated small quantity losses which are difficult to detect using the control chart approach.

6. The calculations involved can be done quickly and can be easily implemented on a sma!l computer. Thus, the technique of !alman Filtering can be used as part of an on-line information gathering and analysis system.

To develop a model of the MBA let:

$I(t)=$ true on-hand inventory at the beginning of period $t$,

$L(t)=$ true material loss in period $t$,

$U(t)=$ true net input to the $M B A$ in period $t$. 
The mass balance equations for the MBA are:

$$
I(t)=I(t-1)+U(t-1)-L(t-1) \cdot C
$$

One may hypothesize various loss scenarios:

1. Constant loss:

$$
L(t)=L(t-1) \text {. }
$$

2. Fraction of on-hand inventory loss (losses are proportional to the on-hand inventory, the known constant of proportionality being $B)$ :

$$
L(t)=\beta I(t-1) \quad 0 \leq \underline{8} \leq 1 .
$$

3. Random loss:

$$
\begin{gathered}
I(t)=I(t-1)+U(t-1)-[L(t-1)+\delta(t-1)] \\
L(t)=L(t-1)
\end{gathered}
$$

where

$$
\begin{aligned}
L(t) & =\text { unknown mean loss } \\
\varepsilon(t) & =\text { zero mean random diversion } \\
\sigma_{\delta}^{i} & =\text { known va:-iance of } \delta(t) .
\end{aligned}
$$

\footnotetext{
The variables $I(t), L(t)$, and $U(t)$ represent the true values of the observed quantities $Y(t), X(t)$, and $W(t)$ which were used in equation (1). The observed quantities are assumed to be the true quantities plus additive noise.
} 
In general, a linear system nodel can be written in matrix form as:

$$
\underline{x}(t)=\left[\begin{array}{c}
I(t) \\
\\
i, t)
\end{array}\right]=\left[\begin{array}{ll}
1 & -1 \\
a_{21} & a_{22}
\end{array}\right]\left[\begin{array}{c}
I(t-1) \\
I(t-1)
\end{array}\right]+\left[\begin{array}{c}
U(t-1) \\
\\
0
\end{array}\right]+\left[\begin{array}{c}
\delta_{1}(t-1) \\
\delta_{2}(t-1)
\end{array}\right] .
$$

The various diversion scenarios are modeled by sperifying $a_{21}$ and $a_{22}$. For a constant diversion, $a_{21}=0$, and $a_{22}=1$. For a fraction of on-hand inventory diversion $a_{21}=\beta$ and $a_{22}=0$. For a random diversion, $a_{21}=0$, and $a_{22}=i$. The error term, $\delta(t-1)$, is used not only to model random diversion, but is a?so used to model measurement errors in the net input. In all cases $Q(t)$ denotes the known covariance matrix of the vector $\underline{c}^{\top}(t)=\left[\delta_{1}(t), \delta_{2}(t)\right]$. Thus $C_{11}(t)$ is the variance of net input measurement error plus the variance of losses. Ideally $C_{22}(t)$ should be zero. In practice, better results are obtained if $C_{22}(t)$ is set equal to a small quantity. Equation (23) gives a precise model of the system state, $\underline{x}(t)$. However, as noti:d earlier, one does not observe $\underline{X}(t)$. Rather, one observes a noisy version of $I(t), Y(t)$. This situation can be modeled by adding an observation equation:

$$
Y(t)=\underline{H} \underline{X}(t)+V(t)
$$

where $\underline{H}=(1,0)$ and $V(t)$ is a zero mean random variable with known variance, $R(t)$.

The Kalman Filter [7], [] $],[9],[10]$, and [11] is a technique for o'staining an estimate of $\underline{x}(t)$ for a linear system of the form of (23) and (24). Specizically, the equation: which define the Kalman Filter are: 


$$
\begin{aligned}
\underline{P}(t)= & \underline{A}(t-1) \underline{G}(t-1) \underline{A}(t-1)^{T}+\underline{Q}(t-1) \\
\underline{K}(t)= & P(t) \underline{H}(t)^{T}\left[\underline{H}(t) \underline{P}(t) \underline{H}(t)^{T}+R(t)\right]^{-:} \\
\underline{\hat{X}}(t)= & \underline{A}(t-1) \underline{\hat{X}}(t-1)+\underline{U}(t-1) \\
& +\underline{K}(t)[Y(t)-\underline{H}(t) \underline{H}(t-1) \underline{\hat{X}}(t-1)+\underline{U}(t-1)\}] \\
& \underline{G}(t)=\underline{P}(t)-\underline{K}(t) \underline{H}(t) \underline{P}(t) .
\end{aligned}
$$

The vector $\underline{\hat{x}}(t)$ is the estimate of $\underline{x}(t)$ produced by the Kalman Filter. The Kalman Filter (equations $(25)-(28)$ ) calculates a gain $\underline{K}(t)$ which is applied to the observation error, $[Y(t)-H(t)\{\underline{A}(t-1) \underline{\hat{X}}(t-1)+U(t-1)\}]$, to produce a new state estimate, $\underline{\hat{x}}(t)$. Using $E()$ to denote expectation and $\underline{e}(t)$ to denote the error, $\underline{\hat{x}}(t)-\underline{x}(t)$, the choice of $\underline{x}(t)$ is such that the trace of $E\left[\underline{e}(t) \underline{e}(t)^{\top}\right]$ is minimized. Matrices $\underline{P}(t)$ and $\underline{G}(t)$ are error covariance matrices. $\underline{P}(t)$ is the error covariance matrix of $\underline{e}(t)$ given $\{Y(t): j=1,2, \ldots, t-1\}$ :

$$
\underline{P}(t)=E\left\{\underline{e}(t) \underline{e}(t)^{\top} \mid Y(1), \ldots, Y(t-1)\right\} .
$$

The matrix $\underline{G}(t) \vdots s$ the error covariance matrix given $\{Y(j): J=1,2, \ldots, t\}$ :

$$
\underline{G}(t)=E\left[\underline{e}(t) \underline{e}\left(t j^{\top} \mid Y(1), \ldots, Y(t)\right] .\right.
$$

Thus, $\underline{P}(t)$ is the error-covariance matrix before receipt of the observation at time $t$, while $G(t)$ is the error-covariance matrix after receipt of the $t^{\text {th }}$ observation. The error-covariance matrix of $\underline{G}(t)$ can be used to determine limits of error. For example, two-sigma control limits are of the form:

$$
L(t) \pm 2 \sigma_{22}(t)
$$


The filter equations are initialized with boundary conditions $\mathbf{G}(0)$ and $\hat{x}(0)$. Theoretical considerations dictate that $\underline{G}(0)$ be any positive definite matrix and $\underline{\hat{x}}(0)$ be an unbiased estimate of $\underline{x}(t)$. Practical considerations dictate the choice of these boundary conditions. It can be shown that $\underline{G}(t)$ approaches the true error covariance matrix and $\underline{\hat{X}}(t)$ approaches $\because(t)$, the rate of convergence being affected by $\underline{G}(0)$ and $\underline{\hat{x}}(0)$. Since $\underline{G}(t)$ depends upon $\underline{G}(0)$ and limits of error depend on $\underline{G}(t), \underline{G}(0)$ can be expected to affect limits of error if (31) is used. The proper choice of $\underline{G}(0)$ and $\hat{x}(0)$ is still in question and renains a subject for further research.

It should also be noted that in applying the Kalman Filter, equations (25), (26), and (28) can be pre-computed. The sequer.ce of optimai $\underline{k}(t)$ values (and $\underline{G}(t)$ if $(23)$ is used) can be pre-calculated and stored. Equation (27) may then be used to produce the cptimal estimates of $\underline{x}(t)$. The roiatively few arithmetic calculations required by (27) and the limited nomory requirements make the Kalman filter ideally suited for an on-line computer system employing a small memory mini-computer.

Figure? shows the flow chart used in implementing the Kalman Filter eyuations. 


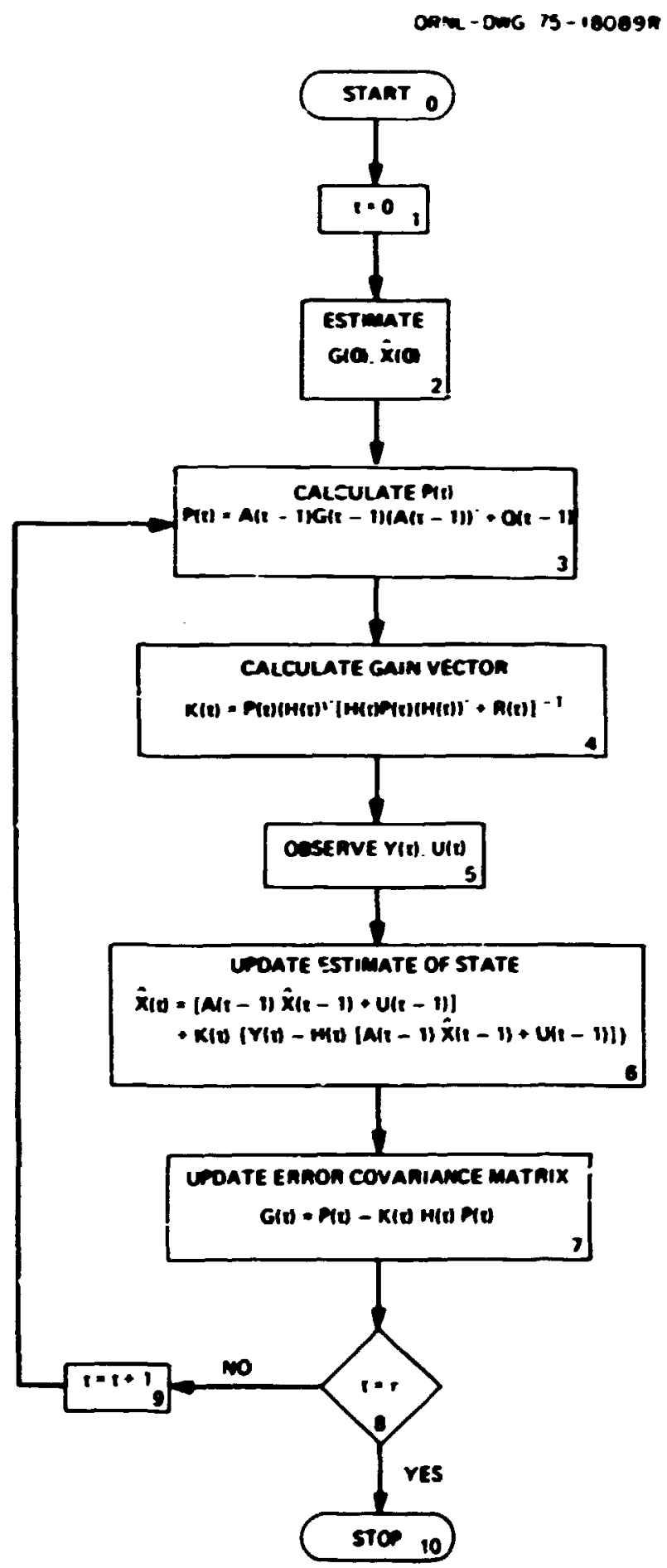

Fig. 1. Flow Chart of the Kalman Filter. 


\section{EXAMPLES}

To test the Kalman Filter approach, several simulations were conducted. Table 1 shows the data for the simulations. The Kalman Filter was initialized with an estimate of the state $\underline{\hat{x}}(0)$ and an estimate of the error covariance matrix $\mathrm{G}(0)$. In all simulations the initial state estimate for on-hand inventory (first element of $\underline{\hat{x}}(0)$ was taken to be 2207. The initial estimate of material lesses (second element of $\underline{\hat{x}}(0)$ ) was zero. This choice of material loss required the filter to correct its initial estimate zero loss and converge to the true material loss.

Table 1. Data Used in Simulations

\begin{tabular}{lc}
\hline Measurement Error Variance & 69.33 \\
Inventory & 0.10 \\
Net Inputs & {$\left[\begin{array}{ll}10 & 0 \\
0 & 1\end{array}\right]$} \\
Initial Error Covariance Matrix $(\hat{G}(0))$ & 0 \\
Net Inputs & 100 \\
Average Value & $(2207,0)$ \\
Standard Deviation & \\
\hline
\end{tabular}

The simulated MBA used computer-simulated measurement noise which was nomally distributed with zero mean and variances of 69.33 for inventory and 0.1 for net transfers. The 2-2 element of $Q$ was set equal to 0.001 . The net transfers were also generated as normal random variables 
with zero mean and a standard deviation of 100 . Over the long run the net transfers averaged zero but in any one period the net transfers could vary anywhere within the range -300 to +300 (i.e., \pm 3 standard deviations). Figure 2 shows the variation of net input over time for the constant loss simulation discussed below.

The first simulation modeled a constant loss of $1 \mathrm{~kg}$ per period (approximately $\mathbf{0 . 5 \%}$ of the on-hand inventory). Figure 3 shows the standard control chart with MUF and LEMF values. This chart fails to detect the loss of $1 \mathrm{~kg}$ per period (i.e., an accumulated loss of $50 \mathrm{~kg}$ ). In a stable system approximately $5 \%$ of the points will exceed the LEMUF. Figure 4 shows an average CUSUM chart with 2-standard-deviation control limits. This chart is more sensitive to losses than the CUSUM chart. However it is not until period 29 that the losses shown on the average CUSUM become significant.

Figure 5 shows the Kalman Filter estimate of material loss along with 2-sigma control limits. The variance used to construct these control limits was obtained from the 2-2 element of the covariance matrix, $\underline{P}(t)$ (Equation 31). This method of loss detection is more sensitive to real material loss than either the standard control chart or the average CUSUM chart. As can be seen, there is an indication of loss in 12 periods. Shortly thereafter the estimate converqes to the true material loss. Finally, Figure 6 shows the Kalman Filter estimate of iriventory and the true inventory values. Note that the estimate is an extremely accurate one. A second simulation of a random loss was also conducted. Losses in this simulation were generated as normal randon variables with a mean of 1.0 and a variance of 0.111 . Figure 7 shows the standard control chart. 
NET MATERIA INPUT FOR CONSTANT LOSS CASE

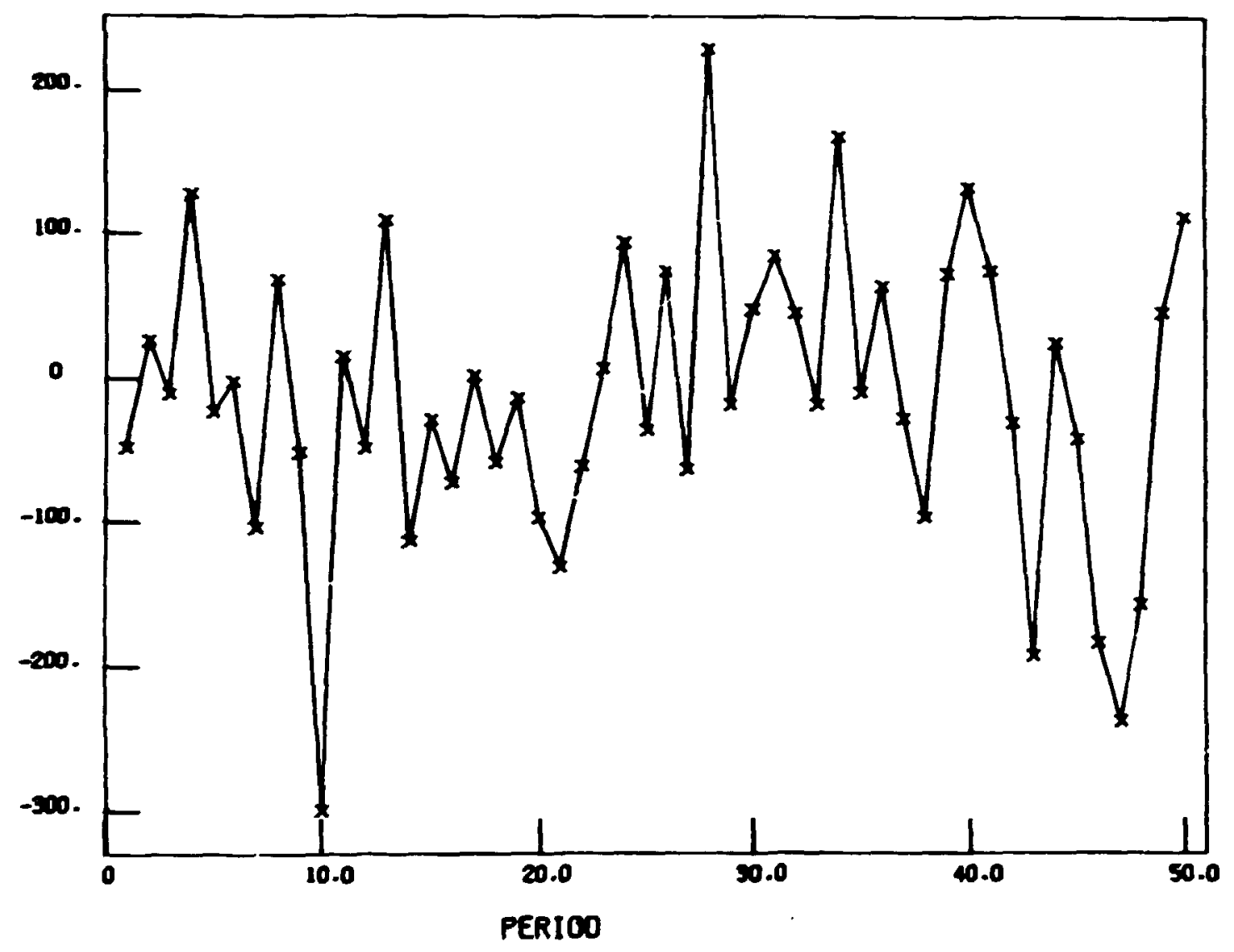

Fig. 2. Net Input Used in Simulation Study for Constant Removal of $1 \mathrm{~kg} /$ period. 


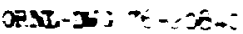

STANOARD CONTROL CHART - CONSTANT LOSS 1 UNIT/PERIOD

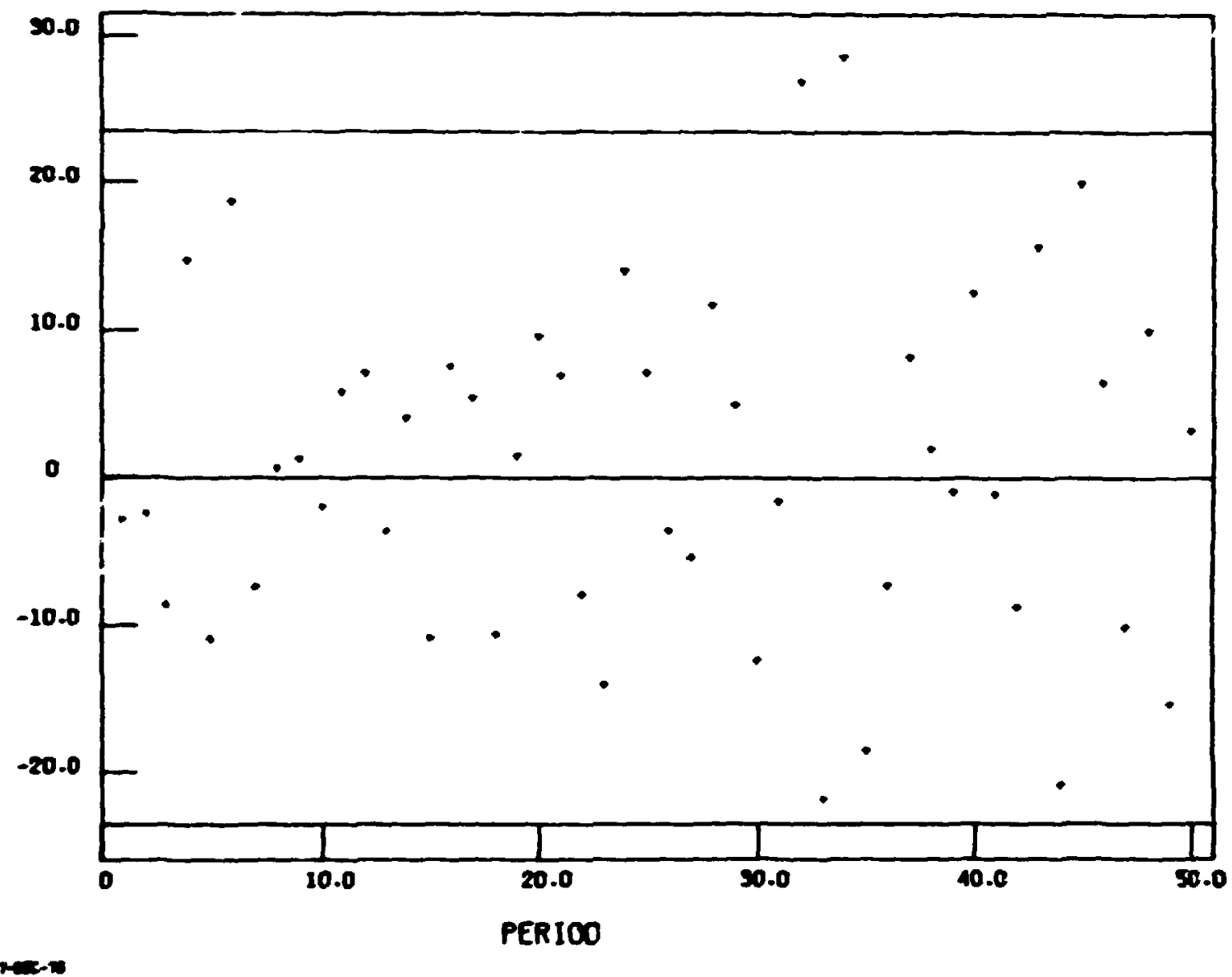

Fig. 3. Standard Control Chart for Simulation Study with a Constant Loss of 1 Unit/period. 
$3 x-2 x ;-\cdots$

AVERACE CUSUM CHART - CONSTANT LOSS 1 UNIT/PERIOD

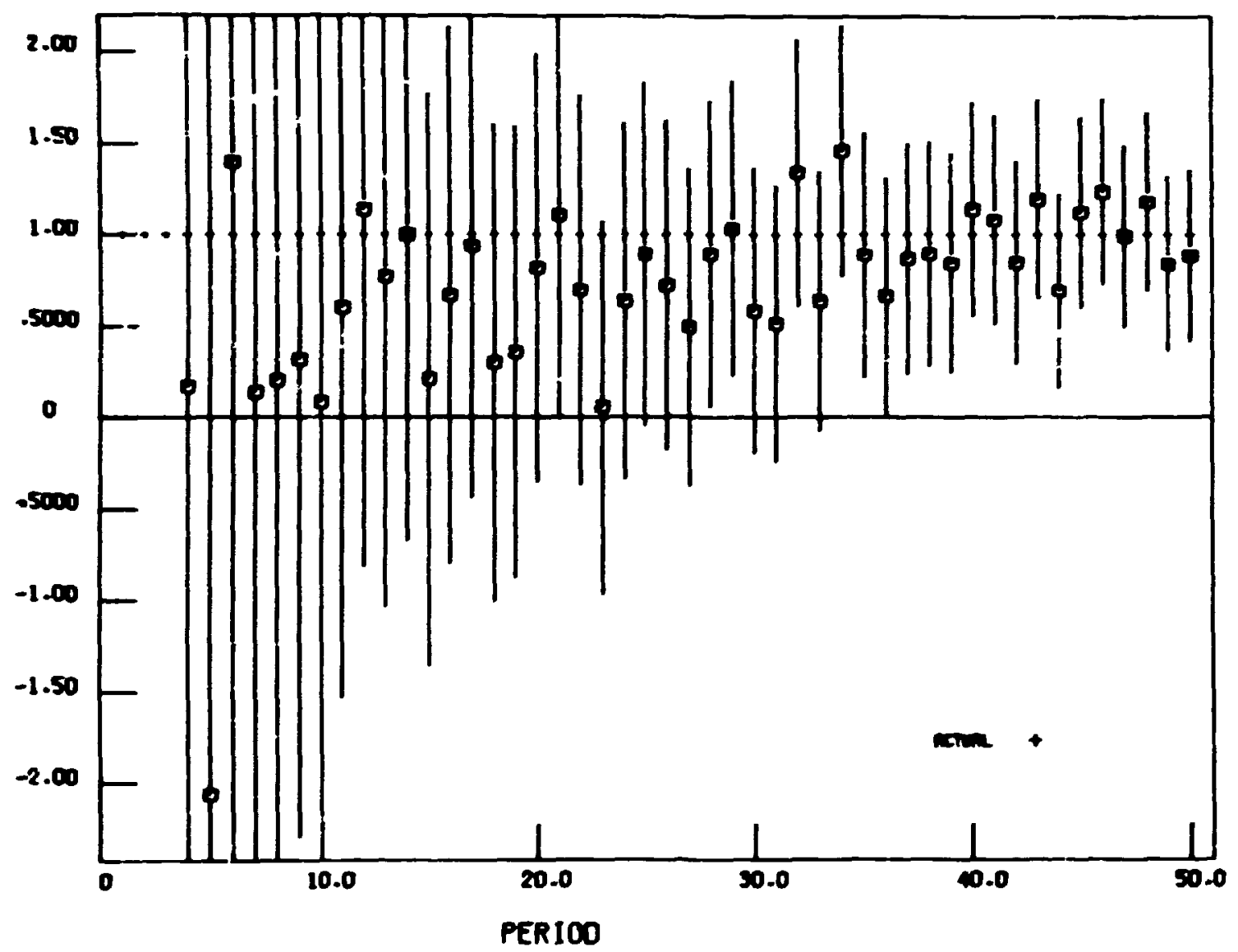

Fig. 4. Average CUSUM Control Chart with Control Limits for a Constant Loss of 1 Unit/period. 
KALMAN FILTER LOSS ESTIMATE - CUNSTANT LOSS 1 LNIT/PERIOO

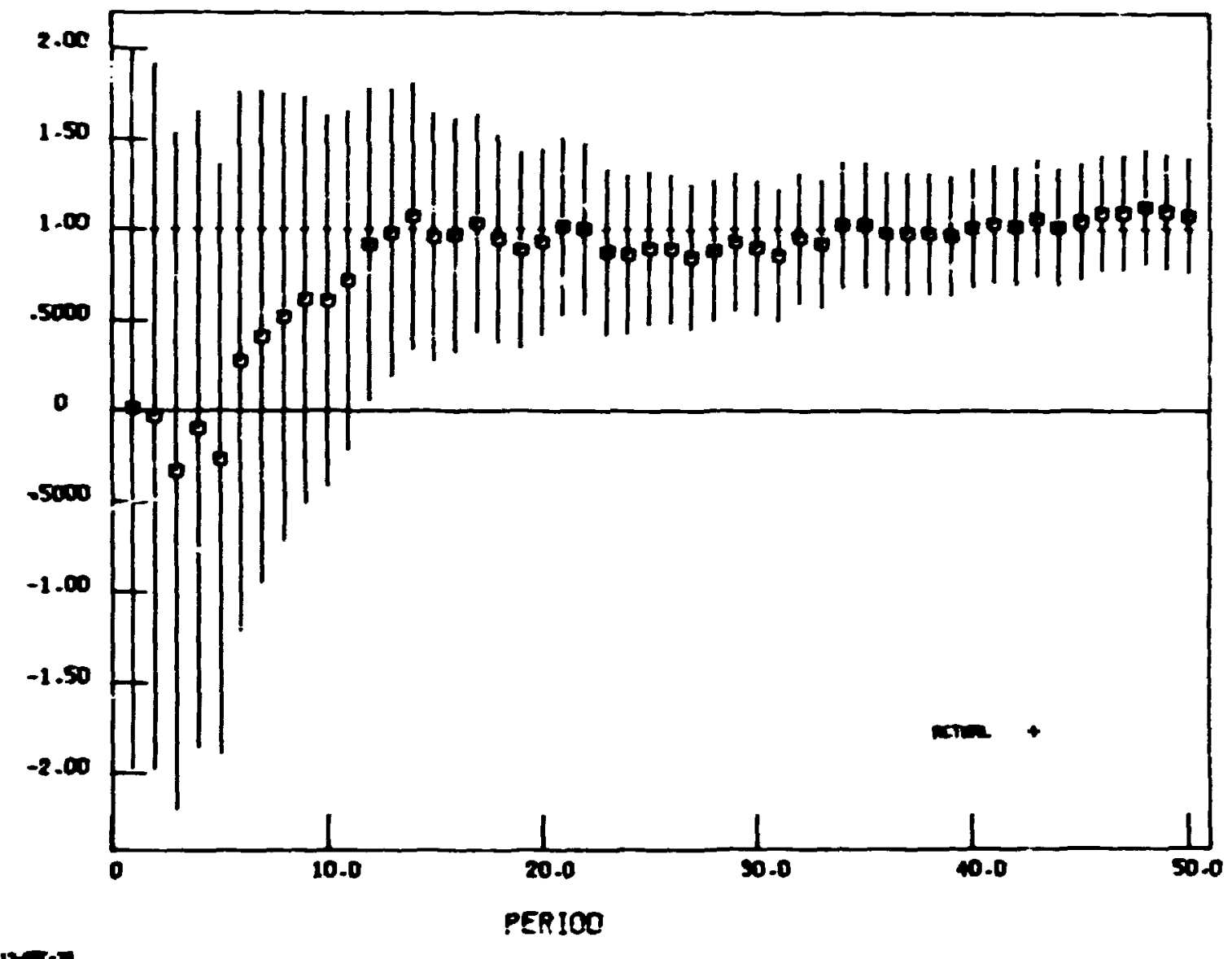

Fig. 5. Kalman Filter Loss Estimate with Control Limits for a Constant Loss of 1 Unit/period. 


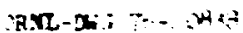

\section{KALMAN FILTER INVENTORY ESTIMATE CONSTANT LOSS MODEL}

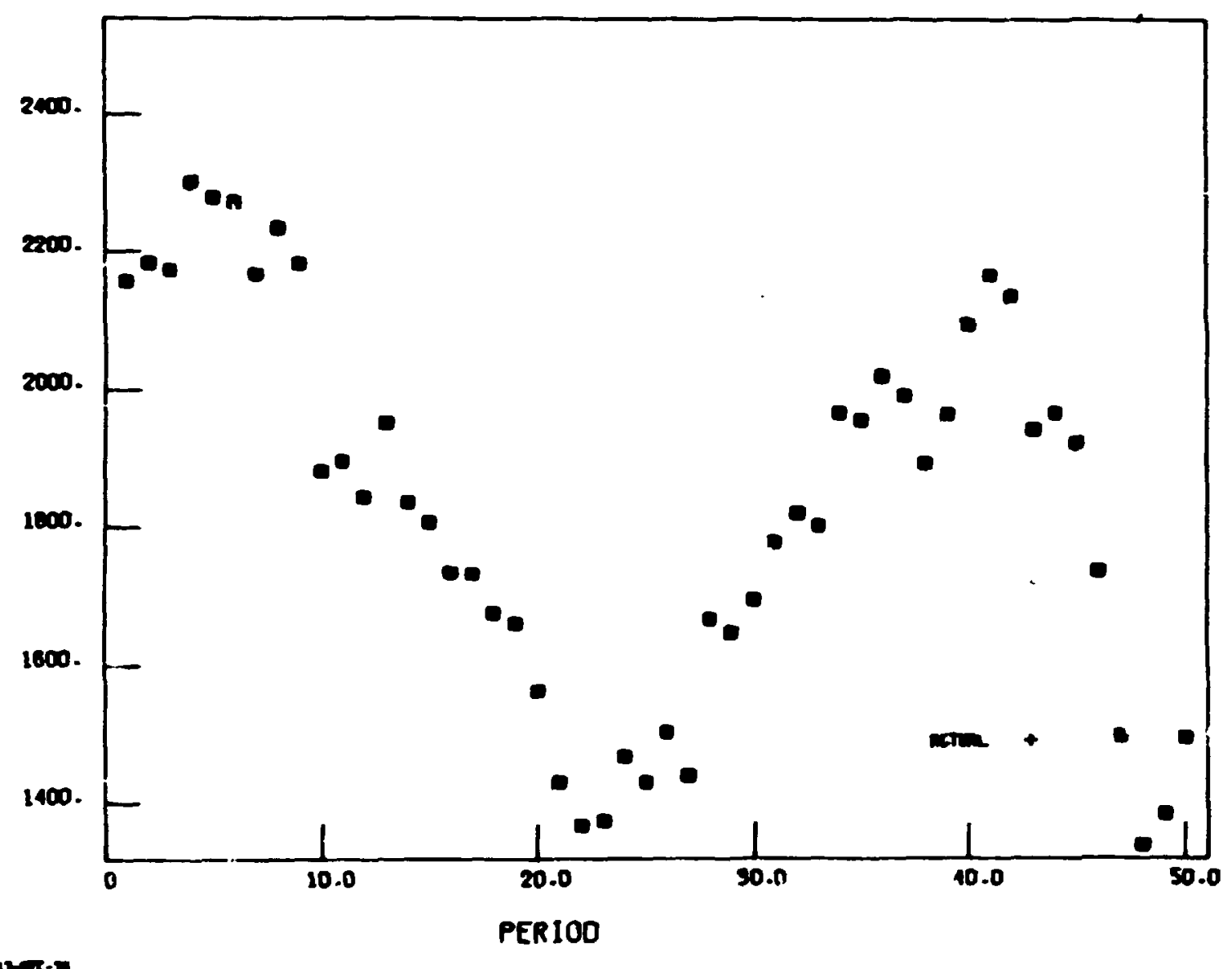

Fig. 6. Kalman Filter Inventory Estimate for Constant Loss of $1 \mathrm{lnnit/period} \mathrm{Case.}$ 
STANOARD CONTRO CHART - RAMDOM LOSS MOOEL

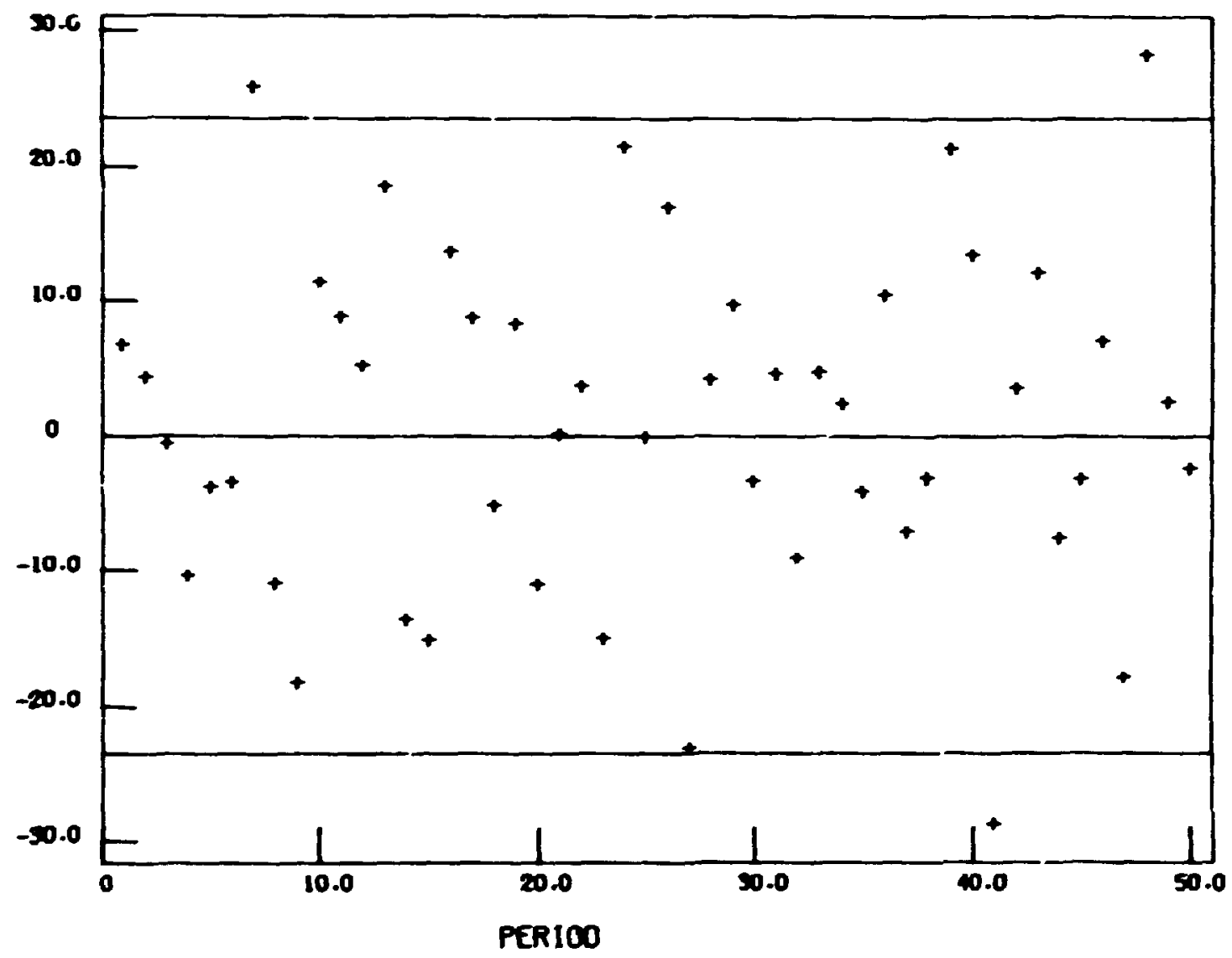

Fig. 7. Standard Control Chart for Simulation Study with a Random Loss with Mean of 1 Unit/period. 
Figure 8 shows the average CUSUM chart and Figure 9 indicates the Kalman Filter estimate. Note that as in the case of the constant loss simulation, the Kalman Filter estimate is a far more sensitive indicator of loss. The Kalman Filter escimate of inventory is shown in Figure 10.

A third simulation shows the results when no material is lost. These results are shown in Figures 11-14. 
AVERACE CUSUM CONTROL CHART - RANDOM LOSS KOOEL

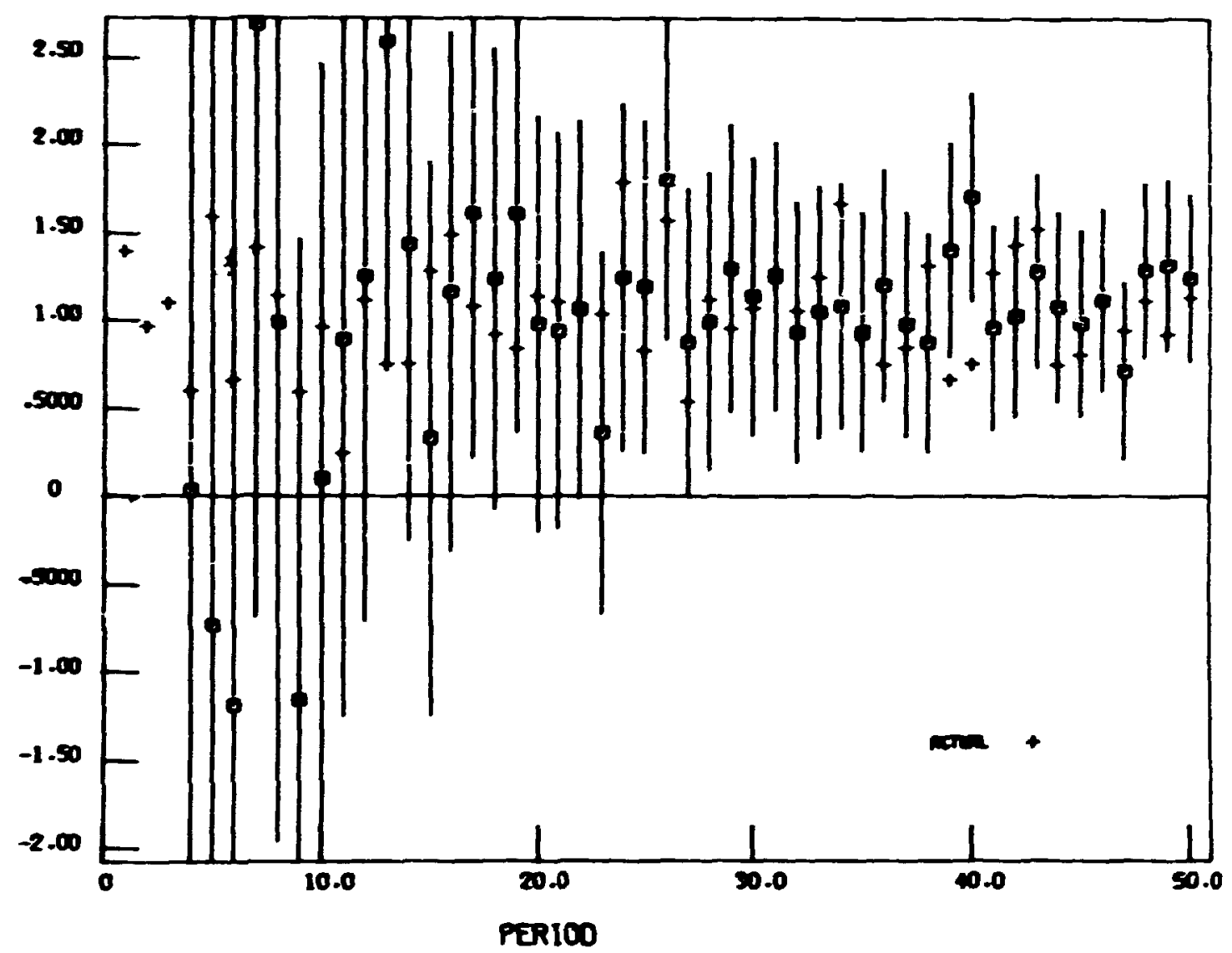

Fig. 8. Average CUSUM Control Chart with Control Limits for Random loss Case with a Mean loss of 1 Unit/period. 
KPLMAN FILITR LOSS ESTIMATE - RPNDON LOSS MOOEL

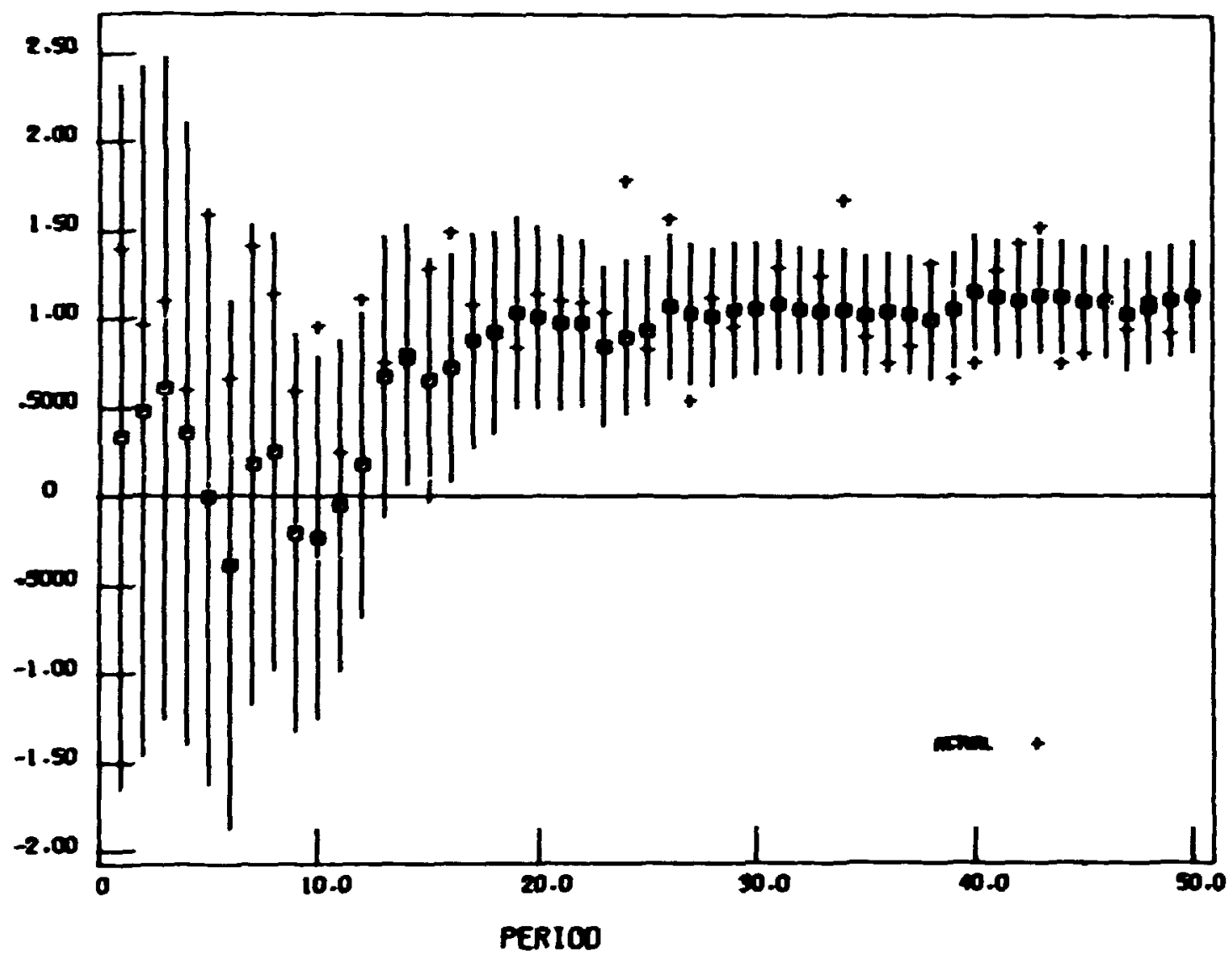

Fig. 9. Kalman Filter Loss Estimate with Control Limits for Random Loss Case with a Mean Loss of 1 Unit/period. 
KRLMPN FILTER INVENTORY ESTIMATE - RPNDON LOSS MODEL

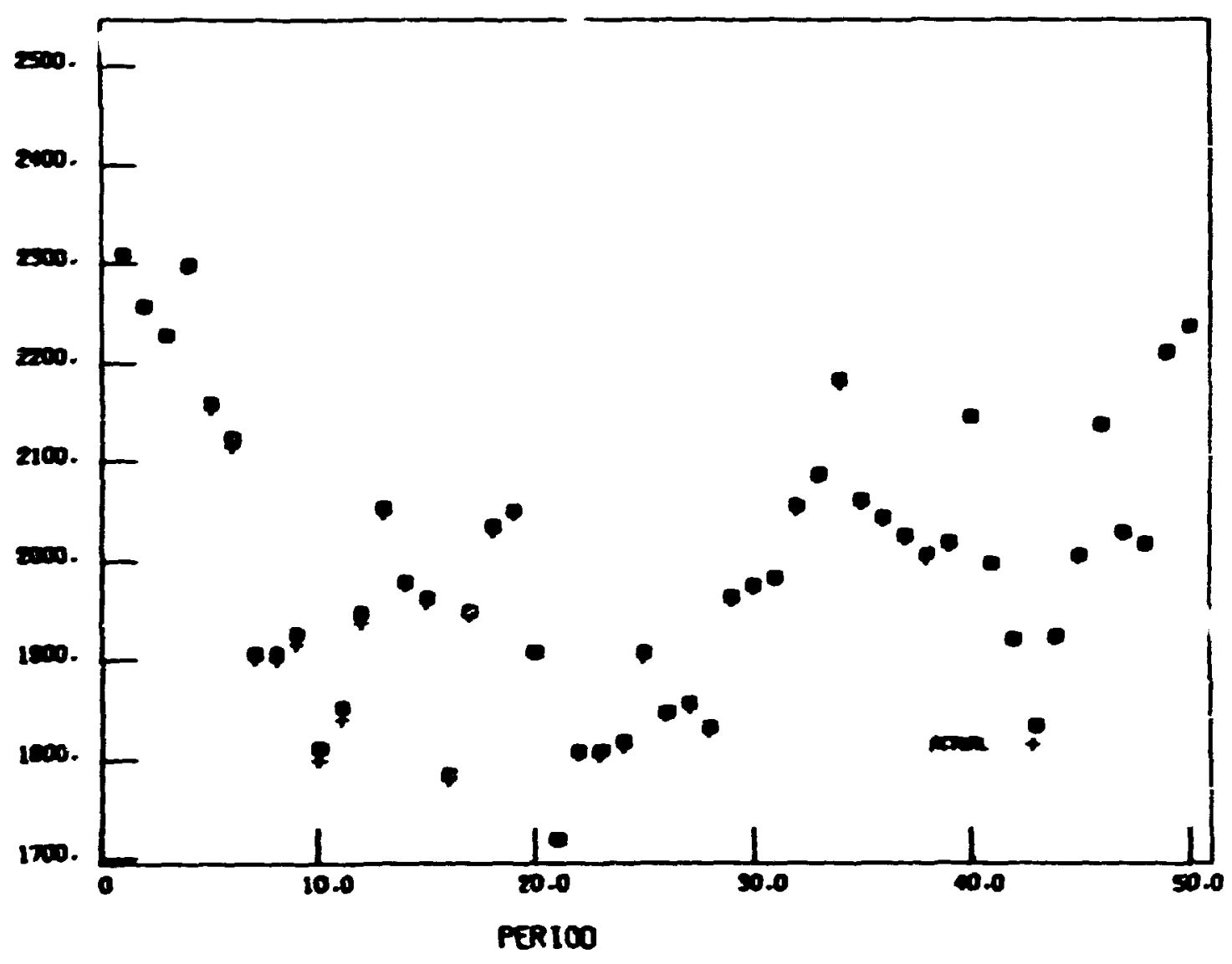

Fig. 10. Kalman Filter Inventory Estimate for Random Loss Case with a Mean Loss of 1 Unit/period. 
STANDARO CONTROL CHART - LOSS = 0 UNIT/PERIOO

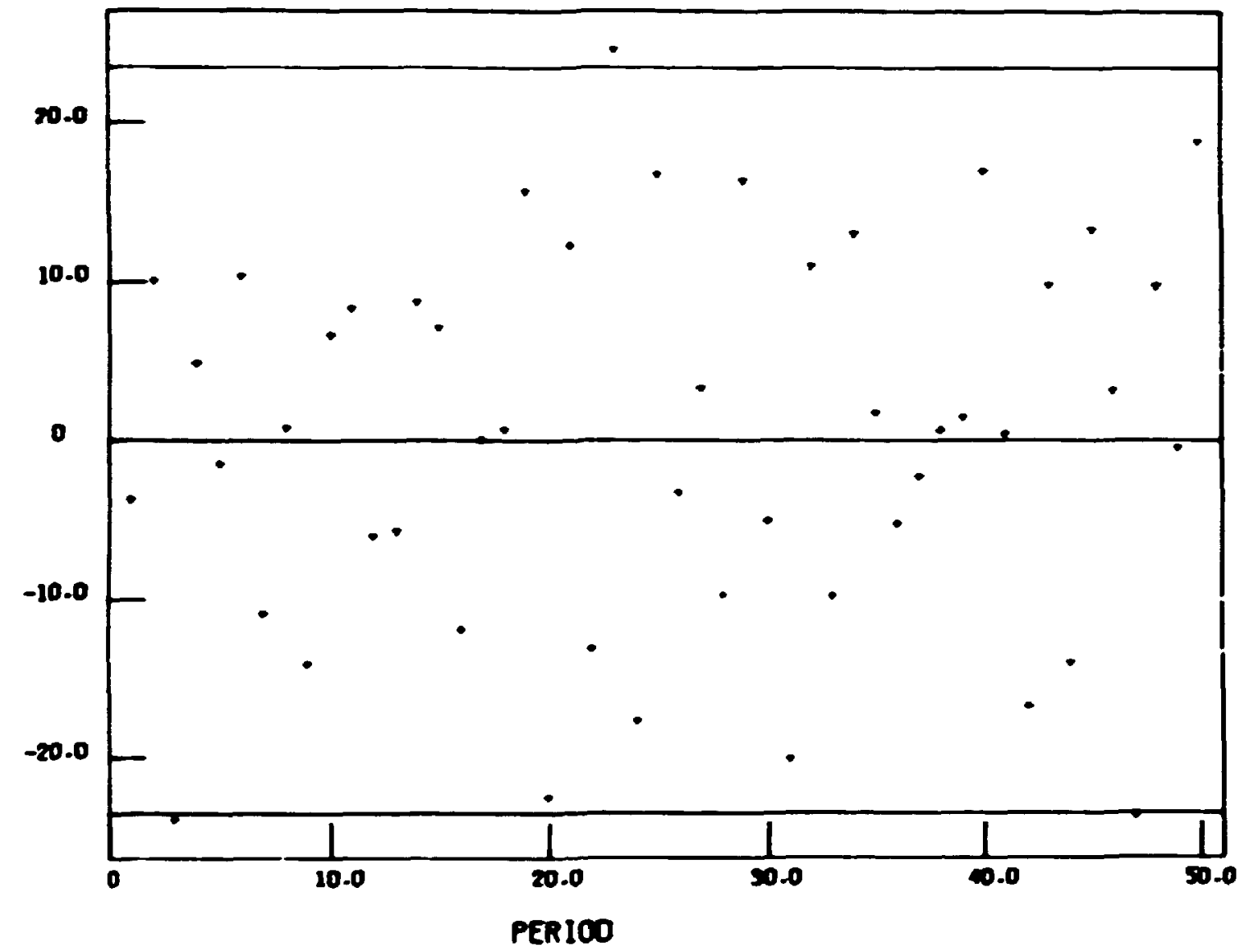

man

Fig. 11. Standard Control Chart for No Loss Case. 
AVERPGE CUSUN CHART - D UNIT/PERIOO LOSS

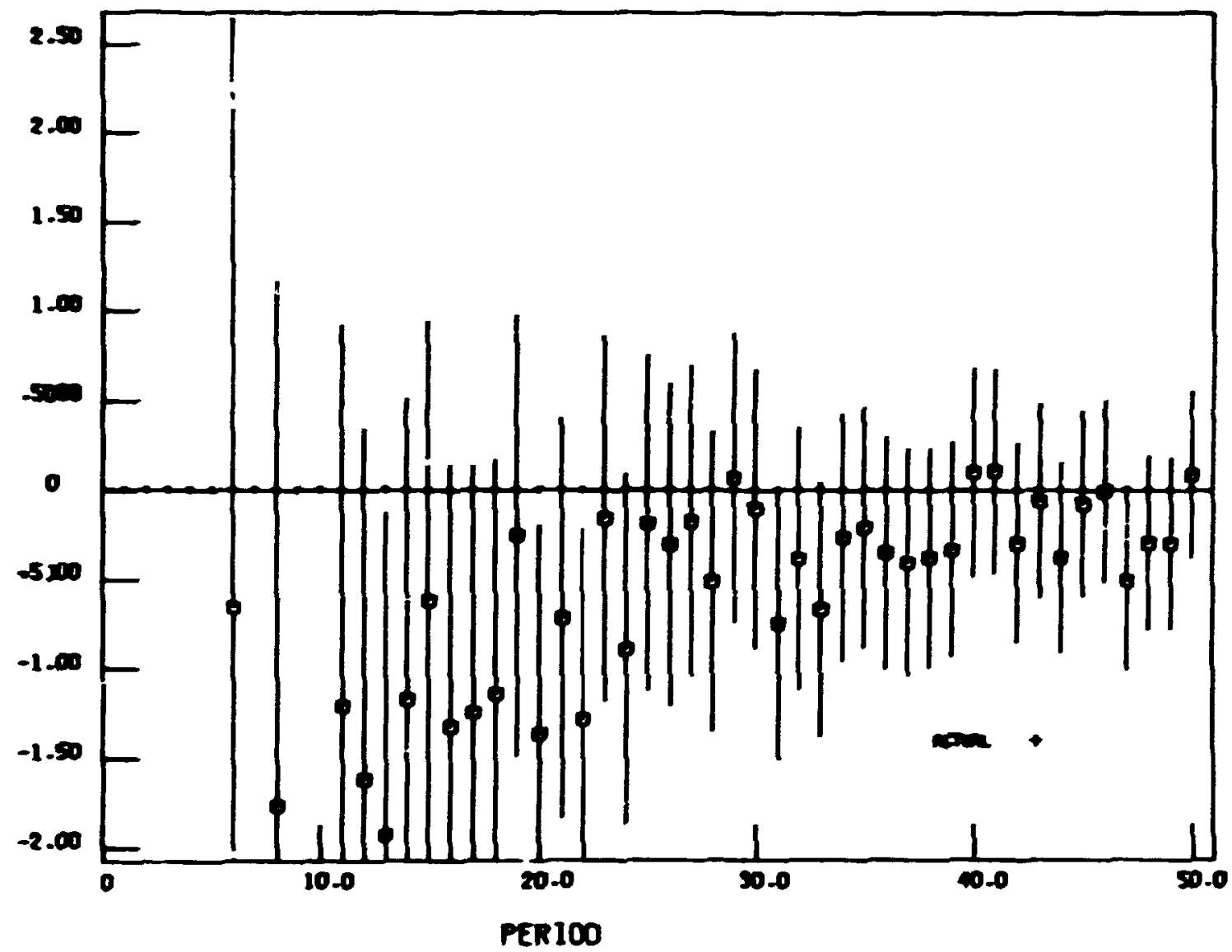

nenso

Fig. 12. Average CuSUM Control Chart with Control Limits for Zero Loss Case. 
$\operatorname{Rx}-36 ;-3 x^{2}=$

KPLMAN FILTER LOSS ESTIMATE - 0 INIT/PERIOO LOSS

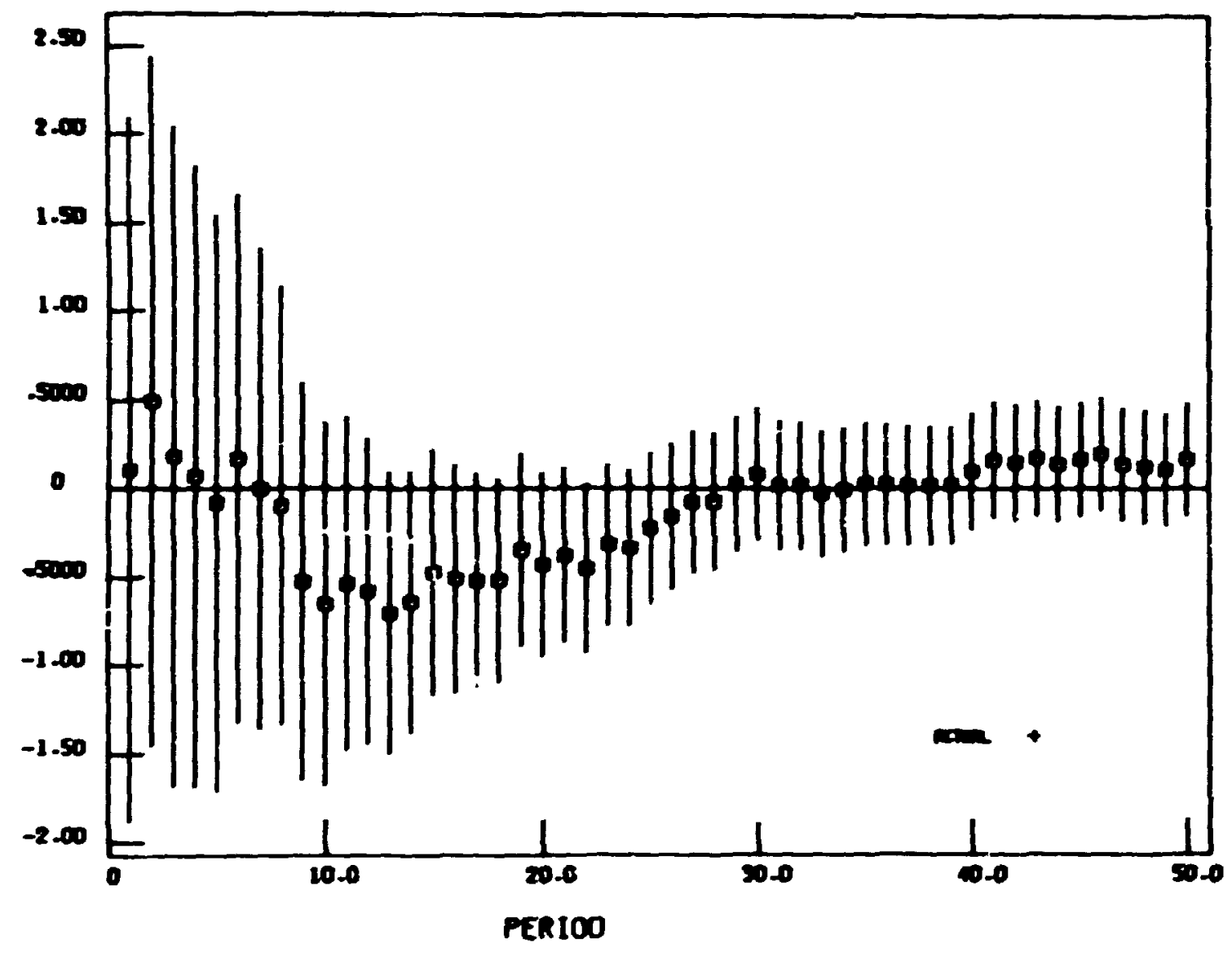

Fig. 13. Kalman Filter Loss Estimate with Control Limits for Zero Loss Case. 


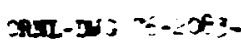

\section{KALMPN FILTER INVENTORY ESTIHATE - 0 UNIT/PERIOO LOSS}

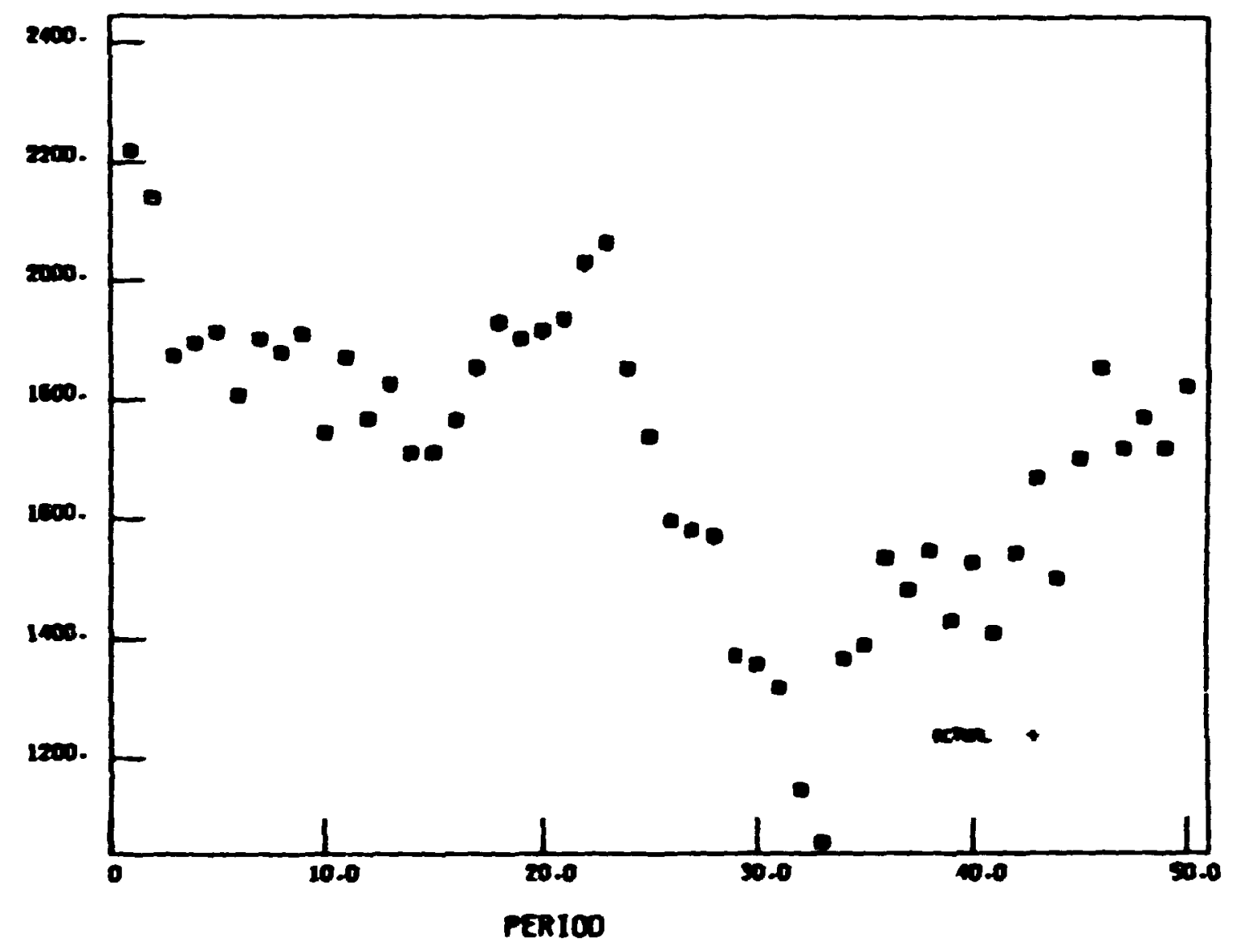

$m$

Fig. 14. Kalman Filter Inventory Estimate far Zero Loss Case. 


\section{CONCLUSIONS}

In many situations nuclear material accountability is limited to the procedure of:

1) Measuring on-hand inventory and material trunsfers.

2) Calculating a book inventory at the end of a period.

3) Calculating a MF from the book inventory and measured inventory.

4) Calculating a LEuff using propagation of error techniques.

5) Comparing MJF to LEMJF.

This procedure will probably indicate a material loss if the loss is larger than the LEMF. The MF-LEuJs approach is limited to analyzing each period's Auf as an isolated data point. The analysis procedure can be enhanced by using the history of MF's and LEMF's and analyzing each period's data in light of the process history. Thus use of control charts particularly the SCC is a logical enhancement of accountability practices. The use of CUSU or average CuSUn charts adds more information in that patterns of small losses are more readily detected.

All of the traditional approaches (SCC, OCC, CUSU, or average CUSUM) are based on analyzing statistics wich are calculated from muF. Referring to equation (1) we see that mW is formed as a linear combination: of three masirexents (two inventory measurements and one net transfer measurenent). The statistic wich we call maf has a variance equal to the sum of the variances of the three random variables used in its calculation (equation 6). The variance of MJF is thus larger than the variance of anj variable wsed in its calruiation. The quantity maf is literally "material unaccounted for" and due to its large variance it is an undesirable statistic to use to detect material losses and should not 
be used as such. Heretofore it was, however, the only indicator known and generally accepted as a measure of material loss.

The research conducted herein shows that the Kalman Filter methodology provides a viable alternative to MUF (and those statistics derived from muf) as a means of detecting material losses. There are several conceptual advantages to using the ralman filter. These include

1) The Kalmari filter estimates true material loss, not observed mif as used in current procedures.

2) Estimates of the on-hand-inventory are produced simultaneously with estimates of material loss. The current procedures use two isolated estimation procedures, one for inventory and another for MUf. Thus current procedures do not account for the interaction of losses and inventory as does the Kalman Filter.

3) The Kalman filter is more sensitive to detecting losses than current procedures.

4) The Kalman filter is ideally suited for use in small comouters or as an integral part of a real-time-information system.

5) The Kalman filter is applicable to the total MBA, mini-MBA's, or unit operations.

In the scenarios studied, the kalman filter proved to be superior to the traditional control chart approacras. Several questions remain for further investigation. These include:

1) The effects of the choice of initial estimates of the state vector and error-covariance matrix on the Kalman filter estimates. 
2) The effect of modeling errors (i.e., using a mudel for a specified scenario when actually another scenario is taking place).

3) How best to use the Kalman Filter in an on-line information system.

4) The effects on estimates of more complex models of the process such as those suggested by Alspach [12]. 


\section{REFERENCES}

1. J. R. Buchanan, "Safeguards Against the Theft or Diversion of Nuclear Materials," Muciear Sxjet:, Vol. 15, No. 5. pp. 513-19 (1974).

2. D. H. Rosenbaun, J. N. Googin, R. M. Jefferson, D. J. Kleitman, and H. D. Sullivan, Special Safeguaris Studi;, unpublished, Oak Fidge National Laboratory Report (1974).

3. J. L. Jaech, Statistical hetrods in huclear Material Controi, USAEC TID-26298, pp. 228-66 (1973).

4. J. L. Jaech, "A New Approach to Calculating LE-MUF," ejo:prazi o: the Institute of luciear Haterials hmajement, Vol. j, No. 2, pp. 14-1? (1972).

5. J. L. Jaech, "Control Charts for MF's," Jouma: o:" tirae Irastituse of Muclear Mcterials isna:ement, Vol. II, No. IV, pp. 16-28 (1974).

6. E. E. Johnson, MUF Control Charts oni LEYUF Gulculations Isin: an Autocorrelation Technigue, Oak Ridge Y-12 Plant Report Y-DJ-16513 (1976).

7. D. H. Pike, G. H. Morrison, and C. H. Holland, "Linear Filtering

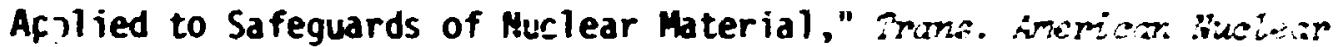
Societ:, Vol. 22, pp. 143-44 (1975).

8. D. H. Pike, G. H. Morrison, and C. H. Holland, "A Comparison of Several Kalman Filter Models for Establishing MUf," Frome. fimericia. Whetrar sorietis, Vol. 23, pp. 267-68 (1976).

9. A. Gelb, Arrlied Optimal Estimatirn, MIT Press, Cambridge, Massachusetts (1974). 
10. R. E. Kalman, "A New Approach to Linear Filterir:g and Prediction

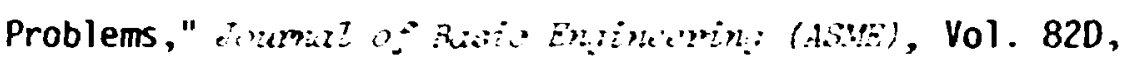
pp. $35-45(1900)$.

11. R. E. Kalman and R. Bucy, "New Results in Linear Filtering and

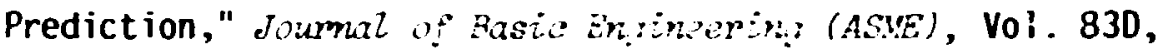
pp. 95-108 (1961).

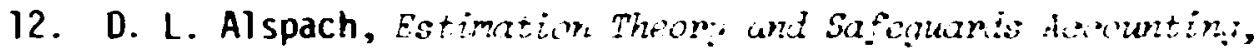
Iniversity of California, Lawrence Livermore Laboratory Report, UCRL-13641 (1975). 
APPENDIX A

Analysis of Control inarts 


\section{Basic Assumptions}

In constructing control charts we are dealing with a noisy observation of on-hand inventory a $i$ the beginning of period $t, Y(t)$ given by:

$$
Y(t)=I(t)+V(t)
$$

where

$$
\begin{aligned}
I(t)= & \text { true on-hand inventory } \\
V(t)= & \text { measurement error. } E[V(t)]=0, \\
& E\left[V^{2}(t)\right]=R(t) .
\end{aligned}
$$

Similarly, net transfers during the period $t$ are assumed to be observed according to the following model:

$$
U(t)=T(t)+H(t)
$$

where

$$
\begin{aligned}
T(t)= & \text { true net transfers }=\Sigma \text { inputs }- \\
& \sum \text { outputs } \\
U(t)= & \text { observed net transfers } \\
H(t)= & \text { observation error. } E[W(t)]=0, \\
& E\left[H^{2}(t)\right]=Q(t) .
\end{aligned}
$$

Observation errors are assumed to be uncorrelated. That is:

$$
\begin{aligned}
& E[V(t), V(t+j)]=0 \text { for all } t \& k \varepsilon\{1,2, \ldots-, \\
& E[W(t), W(t+j)]=0 \text { for all } t \& j \varepsilon\{1,2, \ldots-\}, \\
& E[V(t), W(t+j)]=0 \quad t \varepsilon\{1,2, \ldots-j, j \varepsilon\{0,1, \ldots-\ldots
\end{aligned}
$$


If the book inventory is replaced by the observed inventory at the end of each period, then $\boldsymbol{M ( t )}$ is calculated according to:

$$
M(t)=Y(t)+U(t)-Y(t+1)
$$

The variable $M(t)$ is known as MUF (material unaccounted for) or BPID (Book - Physical Inventory Difference).

Substituting (1) and (2) into (6):

$$
M(t)=[I(t)+T(t)-I(t+1)]+V(t)+W(t)-V(t+1) \cdot \quad(A-7)
$$

Recognizing that true loss, $L(t)$ is

$$
L(\because)=I(t)+T(t)-I(t+1),
$$

it follows that $M(t)$ can be written as:

$$
M(t)=L(t)+V(t)+W(t)-V(t+1)
$$

Equation (9) is more convenient than any of the other equations for determining the moments of $M(t)$.

In particular, we shall be concerned with dete mining the mean and variance of $M(t)$ as well as the mean and variance of other statistics constructed from $M(t)$. 


\section{Analysis of MUF}

At regular intervals it is necessary to 1) measure the inventory $Y(t+1), 2)$ measure the net transfers $U(t)$, and 3) use (6) to calculate $M(t)$.

From (9) and the assumptions on measurement noise it follows that:

$$
E[M(t)]=E[L(t)]
$$

and

$$
\begin{aligned}
\operatorname{Var}[M(t)] & =E[M(t)-E(L(t))]^{2} \\
& =E\left[M^{2}(t)\right]-E^{2}[L(t)] \\
& =E\left[L^{2}(t)\right]+E\left[V^{2}(t)\right]+E\left[V^{2}(t+1)\right]-E^{2}[L(t)]+E\left[W^{2}(t)\right] \\
\operatorname{Var}[M(t)] & =\operatorname{Var}[L(t)]+R(t)+Q(t)+R(t+1) .
\end{aligned}
$$

Equation (11) is used to calculate the "limits of error for MUF" (LEMUF). LEMUF is usually defined to be twice the standard deviation of $M(t)$ assuming $L(t)=0$ :

$$
\text { LEMUF }=2 \sqrt{R(t)+Q(t)+R(t+1)}
$$

Under the conditions that measurement errors are constant from period to period:

$$
\text { LEMUF }=2 \sqrt{2 R+Q}
$$

It has been observed that successive MUFs exhibit correlation. To see this, note that 


$$
\begin{aligned}
\operatorname{Cov} & {[M(t), M(t+i)]=E[M(t) M(t+1)]-E[M(t)] E[M(t+1)] } \\
& =E[(L(t)+V(t)+H(t)-V(t+1))(L(t+1)+V(t+1)+M(t+1)-V(t+2))] \\
& -E[M(t)] E[M(t+1)] \\
& =E[L(t) L(t+1)]-E[L(t)] E[L(t+1)]-E\left[V^{2}(t+1)\right]
\end{aligned}
$$$$
\operatorname{Cov}[M(t), M(t+1)]=\operatorname{Cov}[L(t), L(t+1)]-R(t+1) .
$$

Under the conditions of no losses or constant losses the serial correlation coefficient of lag 1 at time $t, \rho_{1}(t)$ is:

$$
p_{1}(t)=\frac{-R(t+1)}{\sqrt{\operatorname{Var}(M(t))} \sqrt{\operatorname{Var}(M(t+1)}} .
$$

Using the same analysis technique, it can be shown that non-successive MUFS are correlated if, and oniy if, real losses are correlated. In particular:

$$
\operatorname{Cov}[M(t), M(t+i)]=\operatorname{Cov}[L(t), L(t+i)] \quad t \in\{1,2-\cdots\} \text { i }\{2,3-\cdots\}
$$

If true losses are zero (or constant)

$$
\rho_{1}(t)=0 \quad i \in\{2,3, \cdots\}
$$

It is thus possible to rnalyze MUF in several ways. These methods of analysis include 1) testing each individual $M(t)$ against the LEHUF to determine if a non-zero $M(t)$ is significantly different from zero; 2) constructing control charts using the history, $\{M(i): i=1,2,---i\}$, and analyzing "runs"; 3 ) calculating the auto-correlation function, $\rho_{\mathfrak{j}}$, and testing $\rho_{i}(i \geq 2)$ to see $i f$ they are significantly different from zero. 


\section{Differences}

It has been suggested that differences be analyzed.

$$
D(t)=\left[M(t)-p_{1} M(t-1)\right]-\left[E[M(t)]-p_{1} E[M(t-1)]\right]
$$

It can be easily shown that $E[D(t)]=0$.

The variance of $D(t)$ is:

$$
\begin{aligned}
\operatorname{Var}[J(t)]= & E\left[D^{2}(t)\right]=E\left[M^{2}(t)\right]-E^{2}[M(t)] \\
& +\rho^{2} E\left[M^{2}(t-1)\right]-\rho^{2} E^{2}[M(t-1)]-2 \rho \operatorname{Cov}[M(t-1), M(t)]
\end{aligned}
$$

$\operatorname{Var}[D(t)]=\operatorname{Var}[M(t)]+\rho^{2} \operatorname{Var}[M(t-1)]-20 \operatorname{Cov}[M(t-1), M(t)] \cdot(A-18)$

Using equations (11) and (13):

$$
\begin{aligned}
\operatorname{Var}[D(t)]= & \operatorname{Var}[L(t)-\rho L(t-1)]+(1+\rho)^{2} R(t)+\rho^{2} R(t-1)+R(t+1) \\
& +Q(t)+\rho^{2} Q(t-1) .
\end{aligned}
$$

Jaech [3] has suggested estimating $C, E[M(t)]$, and $E[M(t-1)]$ using the history of MUFS, $\{M(i): i=\varepsilon l, 2,-\cdots, t]$.

Assuming zero losses, one can calculate a limit of error for differences (LEDIF)

$$
\text { LEDIF }=2 \sqrt{(1+\rho)^{2} R(t)+\rho^{2} R(t-1)+R(t+1)+Q(t)+\rho^{2} Q(t-1)} .
$$

Assuming the measurement variances do not vary with time:

$$
\text { LEDIF }=2 \sqrt{2\left(1+\rho+\rho^{2}\right) R+\left(1+\rho^{2}\right) Q}
$$

period If $p$ is Small, LED:F $\simeq$ LEMUF 
CUSUNS

Cre can effectively make use of the process history by calculating cumlative sumations (so called CUSUMS). The CUSUn is defined by

$$
Z(t)=\sum_{i=1}^{t} M(i) .
$$

The mean value is

$$
E[Z(t)]=\sum_{i=1}^{t} E[M(i)]=\sum_{i=1}^{t} E[L(i)] .
$$

Note that if $L(i)$ is a constant, $L$, for each period $i$ then

$$
E[z(t)]=t L
$$

Equation (22a) shows that the CUSUM should be capable of detecting small losses which occur regularly over a long period of time.

The variance of $Z(t)$ can be found. Note that since $M(1)=Z(1)$,

$$
\operatorname{Var}[Z(1)]=\operatorname{Var}[L(1)]+R(1)+R(2)+Q(1)
$$

Since $\quad Z(2)=M(1)+M(2)$ :

$$
\begin{aligned}
\operatorname{Var}[Z(2)] & =\operatorname{Var}[M(1)]+\operatorname{Var}[M(2)]+2 \operatorname{Cov}[M(1), M(2)] \\
& =\operatorname{Var}[L(1)]+R(1)+R(2)+Q(1) \\
& +\operatorname{Var}[L(2)]+R(2)+R(3)+Q(2) \\
& +2 \operatorname{Cov}[L(1), L(2)]-2 R(2) \\
& =\operatorname{Var}[L(1)+L(2)]+R(1)+R(3)+Q(1)+Q(2) .
\end{aligned}
$$


If assume that

$$
\operatorname{Var}[2(t-1)]=\operatorname{Var}\left[\sum_{i=1}^{t-1} L(i) j+R(1)+R(t)+\sum_{i=1}^{t-i} Q(i)\right. \text {. }
$$

then

$$
\begin{aligned}
\operatorname{Var}[Z(t)] & =\operatorname{Var}[Z(t-1)+M(t)]=\operatorname{Var}[Z(t-1)]+\operatorname{Var}[M(t)] \\
& +2 \sum_{i=1}^{t-1}\{E[M(i) M(t)]-E[M(i)] E[M(t)] ; \\
& =\operatorname{Var}[Z(t-1)]+\operatorname{Var}[M(t)]+2 \sum_{i=1}^{t-1} \operatorname{Cov}[M(i) M(t)] .
\end{aligned}
$$

Using equations (13) and (15):

$$
\operatorname{Var}[Z(t)]=\operatorname{Var}[Z(t-1)]+\operatorname{Var}[M(t)]+2 \sum_{i=1}^{t-1} \operatorname{Cov}[L(i) L(t)]-2 R(t)
$$

Substituting (23) into the last equation:

$$
\operatorname{Var}[Z(t)]=\operatorname{Var}\left[\sum_{i=1}^{t} L(i)\right]+R(1)+R(t+1)+\sum_{i=1}^{t} Q(i)
$$

From equation (24), one can calculate the standard deviation of $Z(t)$.

This standard deviation can be used to test for significance of $Z(t)$ just as LEMUF is used to test for significance of $M(t)$. Assuming $L(i)=0$ :

$$
\text { LECUSUM }=2 \sqrt{k(1)+R(t+1)+\sum_{i=1}^{t} Q(i)} .
$$

It is interesting to note that if losses are zero and measurement variances are constant with respect to time:

$$
\operatorname{Var}[Z(t)]=2 R+t Q=\operatorname{Var}[M(r)]+(t-1) Q
$$


Thus for large $t$ :

$$
\text { LECUSIN }=2, \operatorname{Var}[Z(t)] \equiv 2, \overline{\mathrm{t0}}
$$

Thus the limits on the CUSUn chart increase as the square root of $t$. If CUSUM charts are mintained, the control limits will increase with increasing $t$.

In analyzing equation 25 it is apparent that the effectiveness of the CUSU chart lies in that fact that wost of the inventory noise measurement noise is removed, that is, the statistic $Z(t)$ removes the variations in $M(t)$ caused by inventory measurenent errors at periods 2 through $t$ (since $R(2), R(3), \ldots, R(t)$ do not appear in LECUSUM). Hence, the CuSU chart can be expected to be more effective in those cases where variation in muF is caused primarily bj inventory measurement errors. 


\section{Average CUSin}

Rather than analyzing CUSUnS, one can analyze the average CUSUnS.

The average cusun at time $t$ is:

$$
\bar{z}(t)=\frac{1}{t} \sum_{i=1}^{t} L(i)=\frac{1}{t} z(t) .
$$

The mean of $\bar{Z}(t)$ is:

$$
E[Z(t)]=\frac{1}{t} E[Z(t)]=\frac{1}{t} \sum_{i=1}^{t}[L(i)]
$$

The variance of $Z(t)$ is:

$$
\operatorname{Var}[\bar{Z}(t)]=\frac{1}{t^{2}} \operatorname{Var}\left[\sum_{i=1}^{t} L(i)\right]+\frac{1}{t^{2}} R(1)+R(t+1)+\frac{1}{t^{2}} \sum_{i=1}^{t} Q(i) \cdot(A-30)
$$

The limit of error is

$$
\text { LEACUSUM }=\frac{1}{t} \text { LECUSUM }+\frac{2}{t} \sqrt{R(1)+R(t+1)+\sum_{i=1}^{t} Q(i)} .
$$

If measurement errors are constant with respect to time:

$$
\begin{aligned}
\text { LEACUSUM } & =\frac{2}{t} \sqrt{2 R+t Q}, \\
& \cong \frac{2 \sqrt{Q}}{\sqrt{t}} \text { for large } t .
\end{aligned}
$$


APPENDIX B

Derivation of the Kalman Filter Equations 
A derivation sizilar to that of Gelb [o] will be followed. The systen model is of the form:

$$
\underline{x}(t+1)=\underline{A}(t) \underline{x}(t)+\underline{u}(t)+\underline{C}(t)
$$

where

$$
\begin{aligned}
\underline{x}(t) & =\text { the systen state vector at time } t, \\
\underline{A}(t) & =\text { knom state transition matrix, } \\
\underline{U}(t) & =\text { a know control vector, } \\
\underline{C}(t) & =\text { a zero-mean randon vector, } \\
\underline{Q}(t) & =E\left[\underline{C}(i) \underline{C}(t)^{\top}\right]=\text { know covariance of } \underline{C}(t), \\
t & =\text { discrete time point } t \in\{0,1,2, \ldots\} .
\end{aligned}
$$

One does not have perfect knoilledge of the state. Rather, one observes $Y(t)$ where:

$$
\underline{Y}(t)=\underline{H}(t) \underline{X}(t)+V(t)
$$

where

$$
\begin{aligned}
& \underline{Y}(t)=\text { Observation vector }, \\
& \underline{H}(t)=\text { Known observation matrix, } \\
& \underline{V}(t)=\text { Zero-mean random vector, } \\
& \underline{R}(t)=E\left[\underline{V}(t) \underline{V}(t)^{T}\right]=\text { known covariance of } \underline{V}(t) .
\end{aligned}
$$

All random variables are assumed to be uncorrelated serially and with zero cross-correlation. The state estimation problem is to obtain an optimal estimate of $\underline{x}(t)$ which will be denoted by $\underline{\hat{x}}(t)$. One is given $\underline{Y}(t), \underline{U}(t), \underline{A}(t), \underline{H}(t), \underline{R}(t)$ and $\underline{Q}(t)$ for each instant of time $t$ where $t$ varies from 0 to $\hat{T}$. The relation between $\underline{\hat{x}}(t)$ and $\underline{x}(t)$ is: 


$$
\underline{\hat{x}}(t)=\underline{x}(t)+\underline{e}(t)
$$

where

$$
\begin{aligned}
& \underline{\hat{x}}(t)=\text { estimate of the state } \underline{x}(t), \\
& \underline{x}(t)=\text { true state at time } t, \\
& \underline{e}(t)=\text { estimation error. }
\end{aligned}
$$

An optimal estimate is defined as an unbiased linear estimate, $\underline{\hat{x}}(t)$, wilich minimizes the expected length of the squared error vector, $E\left[\underline{e}(t)^{\top} \underline{e}(t)\right]$. 


\section{State Estimates}

If one has available an estimate of the state at time $t-1, \underline{x}(t-1)$, the expected state at time $t$ (prior to the observation at time $t$ ) is given by:

$$
\tilde{x}(t)=\underline{A}(t-1) \underline{\hat{x}}(t-1)+\underline{U}(t-1)+\underline{C}(t-1) .
$$

The class of estimators will be restricted to those of the form:

$$
\hat{x}(t)=\underline{J}(t) \underline{\dot{X}}(t)+\underline{k}(t) \underline{Y}(t) \text {. }
$$

If we denote $\underline{x}(t)$ by:

$$
\underline{\tilde{x}}(t)=\underline{x}(t)+\underline{\tilde{e}}(t),
$$

then equation (5) becomes

$$
\begin{aligned}
\underline{\hat{x}}(t)= & \underline{J}(t) \underline{X}(t)+\underline{J}(t) \underline{\tilde{e}}(t)+\underline{K}(t) \underline{H}(t) \underline{X}(t) \\
& +\underline{K}(t) \underline{V}(t) .
\end{aligned}
$$

Subtracting $\underline{X}(t)$ from both sides of $(7)$ :

$$
\begin{aligned}
\underline{e}(t)= & {[\underline{J}(t)+\underline{K}(t) \underline{H}(t)-\underline{I}] \underline{x}(t)+K(t) \underline{v}(t) } \\
& +\underline{J}(t) \underline{\tilde{e}}(t) .
\end{aligned}
$$

We also know that

$$
\begin{aligned}
\underline{\tilde{e}}(t)= & \underline{\dot{x}}(t)-\underline{x}(t)=A(t-1) \hat{x}(t-1)+U(t-1) \\
& -A(t-1) x(t-1)-U(t-1)-c(t-1) \\
\text { or } \quad & \underline{e}(t)=\underline{A}(t-1) \underline{e}(t-1)-C(t-1) .
\end{aligned}
$$


Subsituting (9) into (8):

$$
\begin{aligned}
\underline{e}(t)= & \underline{[J}(t)+\underline{K}(t) \underline{H}(t)-\underline{I}] \underline{x}(t)+\underline{K}(t) \underline{V}(t) \\
& +\underline{J}(t) \underline{A}(t-1) \underline{e}(t-1)-\underline{J}(t) \underline{C}(t-1) .
\end{aligned}
$$

Taking the expectation of (10) and noting that 1) $E[\underline{Y}(t)]=0$,

2) $E[\underline{c}(t-1)]=0$, and 3) that if $E[\underline{e}(t-1)]=0$, the state estimate will be unbiased if and only if the term in brackets is zero. That is

$$
\underline{J}(t)=\underline{I}-\underline{K}(t) \underline{H}(t)
$$

In summary if we choose

$$
\begin{aligned}
\underline{\hat{x}}(t) & =[I-\underline{K}(t) \underline{H}(t)] \underline{\tilde{X}}(t)+\underline{K}(t) \underline{Y}(t) \\
& =\underline{\tilde{X}}(t)+\underline{K}(t)[\underline{Y}(t)-\underline{H}(t) \underline{\tilde{X}}(t)],
\end{aligned}
$$

and if $\underline{\hat{x}}(0)$ is unbiased, all state estimates using (12) will be unbiased. 


\section{Erroi Covariances}

Tiwo error-covariance matrices are of interest: 1) $\underline{P}(t)=E\left[\underline{\tilde{e}}(t) \underline{\underline{e}}(t)^{\top}\right]$ and 2) $\underline{G}(t)=E\left[\underline{e}(t) \underline{e}(t)^{\top}\right]$. From the definitions of $\underline{\tilde{e}}(t)$ and $\underline{e}(t)$, (equations (6) and (3), respectively), $P(t)$ is the error-covariance matrix of the state prior to receipt of the $t^{\text {th }}$ observation wile $G(t)$ is the error covariance matrix immediately following receipt of the $t^{\text {th }}$ observation. Using equation (9), we see that

$$
\begin{aligned}
\underline{P}(t)= & E\left[\underline{\tilde{e}}(t) \underline{\tilde{e}}(t)^{\top}\right]=\underline{A}(t-1) E\left[\underline{\underline{e}}(t-1) \underline{e}(t-1)^{\top}\right] \underline{A}(t-1)^{T} \\
+ & E\left[\underline{C}(t-1) \underline{C}(t-1)^{\top}\right] \quad \text { or } \\
& \underline{P}(t)=\underline{A}(t-1) \underline{G}(t-1) A(t-1)^{\top}+\underline{Q}(t-1) .
\end{aligned}
$$

From equations (8) and (11):

$$
\begin{aligned}
G(t)= & \underline{E}\left[\underline{e}(t) \underline{e}(t)^{\top}\right]=\underline{K}(t)\left[\underline{R}(t)+\underline{H}(t) \underline{P}(t) \underline{H}(t)^{\top}\right] K(t)^{\top} \\
& +P(t)-K(t) H(t) P(t)-P(t) H(t)^{\top} K(t)^{\top} .
\end{aligned}
$$




\section{Choice of Gain}

The optimal choice of $\underline{K}(t)$ is trat $\underline{K}(i)$ which minimizes

$$
E\left[\underline{e}(t)^{T} \underline{e}(t)\right]=\operatorname{trace}[\underline{e}(t)] .
$$

Noting that:

$$
\frac{d}{d \underline{x}} \text { trace }\left[\underline{X A X}^{\top}\right]=2 \underline{X A} \text { (A Symmetric) }
$$

and

$$
\frac{d}{d \underline{X}} \operatorname{trace}[\underline{X A}]=\frac{d}{d \underline{X}} \text { trace }[\underline{A} \underline{X}]=\underline{A}^{\top}
$$

and using (14):

$$
\begin{aligned}
\left.\frac{d}{d \underline{K}(t)} \operatorname{trace}[\underline{G}, t)\right]= & \underline{2 K}(t)\left[\underline{R}(t)+\underline{H}(t) \underline{P}(t) \underline{H}(t)^{\top}\right] \\
& -2 \underline{P}(t) \underline{H}(t)^{\top}=\underline{0}
\end{aligned}
$$

or

$$
\underline{K}(t)=\underline{P}(t) \underline{H}(t)^{\top}\left[\underline{R}(t)+\underline{H}(t) \underline{P}(t) \underline{H}(t)^{\top}\right] .
$$

The Hessian Matrix is:

$$
\frac{d^{2}}{d \underline{K}(t)^{2}} \operatorname{trace}[\underline{G}(t)]=\left[\underline{R}(t)+\underline{H}(t) \underline{P}(t) \underline{H}(t)^{\top}\right] .
$$

Hence, as long as the Hessian Matrix in 20 is positive definite, the solutior. given by (19) exists and is optimal. The Hessian Matrix will be positive definite if $P(t)$ is positive definite, and the rank of $\underline{H}(t)$ is full. Substituting (19) into (14) it follows that

$$
\underline{G}(t)=[\underline{I}-\underline{K}(t) \underline{H}(t)] \underline{P}(t) \text {. }
$$




\section{Kalman Filter}

Equations (4), (12), (13j, (19), and (21) constitute the iterative procedure known as the $\mathrm{K}_{\mathrm{i}} \mathrm{lman}$ filter. These equations are restated below:

1) Choose $\underline{x}(0)$ and unbiased estimate of $\underline{x}(0)$ and $\underline{\underline{g}}(0)$ a positive definite error covariance matrix. Set $t=1$.

2) State Prediction:

$$
\underline{\tilde{x}}(t)=\underline{A}(t-1) \underline{\hat{x}}(t-1)+\underline{U}(t-1) .
$$

3) Calculate error covariance matrix for state estimate in last step

$$
\underline{P}(t)=\underline{A}(t-1) \underline{G}(t-1) \underline{A}(t-1)^{\top}+\underline{Q}(t-1) .
$$

4) Calculate gain:

$$
K(t)=\underline{P}(t) \underline{H}(t)^{\top}\left[\underline{H}(t) \underline{P}(t) \underline{H}(t)^{\top}+\underline{R}(t)\right]^{-1} .
$$

5) Update state estimate

$$
\underline{\hat{x}}(t)=\underline{\tilde{X}}(t)+\underline{K}(t)[\underline{Y}(t)-\underline{H}(t) \underline{\tilde{X}}(t)] .
$$

6) Calculate error covariance matrix for the state estimate in the last step:

$$
\underline{G}(t)=[\underline{I}-\underline{K}(t) \underline{H}(t)] \underline{P}(t) .
$$


58

State Prediction

If one is interested in forecasting future values of the state vector, the linear system equation (1) can be used iteratively. Given that the most recent state estimate is at time $t$ and using the boundary condition:

$$
\begin{gathered}
\underline{\dot{x}}(t)=\underline{\hat{x}}(t) \\
\underline{x}(t+\tau+1)=\underline{A}(t+\tau) \underline{\bar{x}}(t+\tau)+\underline{U}(t+\tau) \quad i=: 0,1,2, \ldots, \dot{j}
\end{gathered}
$$

where $\underline{x}(t+\tau)$ denotes the forecaster value of the state vector + periods into the future. One can obtain the error covariance matrix for the estimate in (23) by using (9) iteratively:

$$
\begin{aligned}
\underline{G}(t+i+1) & =E\left[\underline{\dot{e}}(t+\tau+1) \underline{e}(t+\tau+1)^{\top}\right] \\
& =\underline{A}(t+\tau) \underline{G}(t+\tau) \underline{A}(t+\tau)^{\top}+\underline{Q}(t+\tau) .
\end{aligned}
$$

